\title{
Simultaneous micro-PIV measurements and real-time control trapping in a cross-slot channel
}

\author{
Farzan Akbaridoust ${ }^{1}$, Jimmy Philip ${ }^{1}$, David R. A. Hill ${ }^{2}$ and Ivan Marusic ${ }^{1}$
}

Received: date / Accepted: date

\begin{abstract}
Here we report novel micro-PIV measurements around micron-sized objects that are trapped at the centre of a stagnation point flow generated in a cross-slow microchannel using real-time control. The method enables one to obtain accurate velocity and strain rate fields around the trapped objects under straining flows. In previous works, it has been assumed that the flow field measured in the absence of the object is the one experienced by the object in the stagnation point flow. However, the results reveal that this need not be the case and typically the strain rates experienced by the objects are higher. Therefore, simultaneously measuring the flow field around a trapped object is needed to accurately estimate the undisturbed strain rate (away from the trapped object). By combining the micro-PIV measurements with an analytical solution by Jeffery (1922), we are able to estimate the velocity and strain rate around the trapped object, thus providing a potential fluidic method for characterising mechanical
\end{abstract}

Farzan Akbaridoust

farzan.akbaridoust@unimelb.edu.au

1 Department of Mechanical Engineering, University of Melbourne, Parkville, Victoria 3010, Australia

2 Department of Chemical and Biomolecular Engineering, University of Melbourne, Parkville, Victoria 3010, Australia

Electronic Supplementary Information (ESI) available: We provide the culture growth and maintenance conditions (ESI section 1); simulation of the control system to trap a target object (section 2); analytical solution of the flow over an ellipsoidal object immersed in a creeping straining flow (section 3); Videos of trapping a single filament of $A$. circinalis (movie $\mathrm{S} 1$ ), and particles flowing around a single filament (movie S2) are also provided. properties of micron-sized materials, which are important in biological and other applications.

Keywords Microfluidics · Microfluidic trap · MicroPIV · Particle Image Velocimetry · Stagnation point · Extensional flow

\section{Introduction}

Hydrodynamic-based microfluidic traps have been widely used to confine and manipulate micron-sized objects in an extensional (stagnation point) flow in cross-slot microchannels. This microfluidic platform is used as a valuable tool in the fields of biology and engineering to carry out single cell analysis and study the dynamics of other micronsized objects (Johnson-Chavarria et al., 2011, 2014; Tanyeri and Schroeder; Tanyeri et al., 2010, 2011). Typically, there are two aspects of the hydrodynamicbased microfluidic trap; first, to control and trap the micron-sized objects in the flow, and second, the characterisations of the flow itself, are studied separately. For trapping, the conventional method involves using a video camera that monitors a micron-sized object in the microfluidic device and adjusting pressure in an onchip membrane valve as a feedback loop to ensure that the object remains trapped at the zero-velocity (stagnation) point of the extensional flow (Tanyeri et al., 2010). The flow characterisation, the other aspect of the platform, is carried out by numerical simulation or using micron-resolution particle image velocimetry (micro-PIV) measurements of the flow inside the microfluidic device, however, with the object absent. In fact, simultaneous trapping of objects and velocity measurements is challenging because both require their own illumination, optics and camera focused on the micron 
sized objects. Consequently, it is usually assumed that the flow field measured in the absence of the object is the one experienced by the object, though this need not be the case depending on the object size relative to the channel dimensions. Most of the efforts on this type of trap have concentrated on improving the ability to confine the objects, which include increasing the trap stiffness and residence time and they have relied on the flow characteristics of the extensional flow with the absence of the target objects. However, in this study, we develop a unique method that enables one to obtain the flow characteristics in-real time while a single target is trapped in the flow. This enables one to calculate more precise information regarding the flow parameters including the strain rate experienced by the trapped objects. We find that trapped objects change the flow. As such, velocity field measured in the absence of the object may not represent the correct flow field in the microfluidic device.

Over the last two decades, advances in microfluidic technology have enabled researchers to trap and immobilise nano- and micron-sized objects such as single micro-particles, cells and macroDNA molecules using different types of microfluidic traps such as magnetic (Gosse and Croquette, 2002; Lee et al., 2004), optical (Ashkin et al., 1986; Grier, 2003; Yang et al., 2009), acoustic (Bernassau et al., 2014; Hertz, 1995; Qiu et al., 2014) and hydrodynamic traps (Tanyeri et al., 2010, 2011). Amongst the traps mentioned, the hydrodynamic trap solely utilises hydrodynamic forces for confinement of a micron-sized object in a flow, whereas the others are dependent on external forces such as magnetic, acoustic and optical. Therefore, a hydrodynamic trap is the best option to conduct micro-biological experiments when similarity of the flow to their natural habitat is required.

The first hydrodynamic trap was in a four-roll mill (Taylor, 1934), which is suitable for trapping millimetre-sized objects. It is not a suitable platform for confining micron-sized objects due to the difficulty of maintaining a fixed location of the stagnation point within one micron (Akbaridoust et al., 2018).

Another apparatus that can produce the same type of flow as Taylor's four-roll mill is a cross-slot channel (schematically shown in figure 1). Cross-slot channels consist of two co-linear converging inlets, and two diverging outlet laminar streams orthogonal to the inlet flows. The two inlet laminar flows meet at the intersection of the cross-junction, where a two-dimensional extensional flow with a zero-velocity (stagnation) point and hyperbolic streamlines are generated. The velocity equations of a pure stagnation point flow are given by $u=\gamma x, v=-\gamma y$, where $\gamma$ is the strain rate of the flow. Ideal straining stagnation point flow has hyperbolic streamlines ( $x y=$ const.) and spatially uniform strain rate $(\gamma=$ const. $)$.

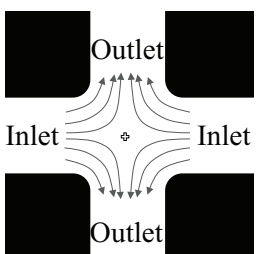

Fig. 1: Schematic of a cross-slot channel.

\subsection{Trapping of objects in cross-slot microchannel}

In the 1990s, with the emergence of microfluidic devices, cross-slot microchannels were used to trap micronsized objects such as DNA molecules in a stagnation point flow. However, this was limited to shortterm confinement of the target (Perkins et al., 1997) as the stagnation point is a semi-equilibrium stable point (Tanyeri et al., 2010). Later, relatively longer confinement of single objects was achieved using humanfacilitated control systems in cross-slot microchannels (Dylla-Spears et al., 2010; Schroeder et al., 2003, 2004; $\mathrm{Xu}$ and Muller, 2011).

Over the last several years, using an image-based active control system, long-term trapping of a single micron-sized object became possible. Schroeder and co-workers (Johnson-Chavarria et al., 2011, 2014; Latinwo et al., 2014; Li et al., 2015; Shenoy et al., 2015; Tanyeri and Schroeder; Tanyeri et al., 2010, 2011) developed an image-based microfluidic hydrodynamic trap in the straining flow of a cross-slot microchannel for high-resolution confinement of single micron-sized objects. To tackle the Brownian motion and to achieve long-term confinement of micron-sized beads, Tanyeri et al. (2010) implemented an on-chip membrane valve (filled with a pressurised gas) on one of the outlets of the cross-junction of the microfluidic device to control the position of the stagnation point. Johnson-Chavarria et al. (2011) demonstrated the fabrication of the double-layer microfluidic device used in this type of microfluidic trap and visualised how to operate the trap. Tanyeri et al. (2011) comprehensively presented the design and implementation of the controlled hydrodynamic trap and characterised the effect of various parameters used in fabrication of the microfluidic device and the dimensions of the device on the time response of the membrane valve. Curtis et al. (2011) simulated the flow and the control system in a 
cross-slot microfluidic device with a considerably lower computational cost in comparison with conventional simulations to propose an apparatus for cell sorting. Johnson-Chavarria et al. (2014) used an adaptive control algorithm to achieve longer trapping and they confined an Escherichia coli cell, to investigate the effect of cell growth environment by observation of the cell growth dynamics, intracellular diffusion proteins and gene expression. The same basic concepts of the trapping were used by Shenoy et al. (2016), in a microfluidic device with more inlets and outlets to produce a double-stagnation point flow to simultaneously trap multiplexed particle manipulation (called a Stokes trap).

Recently, cross-slot microfluidic devices were used as means of single-cell Mechanophenotyping (Cha et al., 2012; Gossett et al., 2012; Henon et al., 2014; Henry et al., 2013). For a high throughput of 2000 cell/s, Gossett et al. (2012) and Henry et al. (2013) applied very high strain rates (about $10^{5} \mathrm{~s}^{-1}$ ) for a high throughput of 2000 cell/s. Using an inertial focusing technique and a high speed camera, they observed and characterised the cell deformability at the cross-slot junction. However, the residence time of the sample was $\mathrm{O}\left(10^{-5} \mathrm{~s}\right)$. The method is therefore not suitable for long-term straining flow exposure to target objects.

1.2 Velocity measurements in cross-slot microchannel

Researchers have measured the velocity field by either tracking a few particles (Tanyeri et al., 2010), or to acquire more precise results, others have measured velocity and strain rate fields using micro-PIV (Akbaridoust, 2017; Akbaridoust et al., 2018; Alicia et al., 2016; Pathak and Hudson, 2006; Ulloa et al., 2014). Some have obtained the flow field in the cross-junction using numerical simulations (Dylla-Spears et al., 2010).

In none of the studies of cross-slot microchannels, micro-PIV measurements have been carried out simultaneously while a micron-sized object is trapped, and all the researchers relied on the velocity strain field of the flow with the absence of the trapped objects. Simultaneous flow measurements while a target object is trapped (presented in this work) is crucial when relatively large objects are trapped (De Loubens et al., 2015; Gossett et al., 2012), as the average flow strain rate is higher than flow strain rate with the absence of the target, due to obstruction from the target object.

\subsection{Present investigation}

In our previous work (Akbaridoust et al., 2018), we systematically measured the velocity fields at relatively high strain rate of up to about $140 \mathrm{~s}^{-1}$ for different flow rates using micro-PIV and fully characterised the flow to identify the uniform strain rate region. In this work, by modifying the image-based active control technique in cross-slot microchannels we trap single objects in the straining flow (hydrodynamic trap), and simultaneously measure the velocity field using micro-PIV around micron-sized objects while being trapped in the stagnation point. This enables one to calculate the stretching and compressing forces on the filament. It also opens up new vistas of characterising mechanical properties of micron-sized objects. No such measurements have been carried out while a micron-sized object was confined at the stagnation point and all previous studies were based on the measurement of the ideal flow with the absence of the trapped object. Here, we trapped and measured the velocity around filaments of Anabaena circinalis (recently renamed Dolichospermum circinale by Wacklin et al. (2009)), of the cyanobacteria species, which is a cause of significant water contamination worldwide (Cook et al., 2010). In addition to A. circinalis, we grew, trapped and exposed the green alga, Dunaliella tertiolecta (the single-celled motile marine species) to demonstrate the capability of the microfluidic trap to confine both motile and non-motile microorganisms and compare the results with the simulation of the microfluidic trap. Although interesting, in this paper we do not focus on the effects of strain rate on the waterborne microorganisms.

\section{Fabrication of microfluidic device}

Double-layer microfluidic devices were fabricated with features shown in figure 2 using soft lithography. The height and width of the channel are 40 and $400 \mu \mathrm{m}$ respectively (an aspect ratio of $\alpha_{r}=h / w=0.1$ ). The fabrication protocol of a double-layer microfluidic device (a cross-slot microchannel with an on-chip membrane valve) is thoroughly detailed in Akbaridoust et al. (2016) and Johnson-Chavarria et al. (2014). The black features in figure 2 indicate the fluidic channel (crossslot microchannel) and the red features indicate the control channel (on-chip membrane valve). The protocol suggested by Johnson-Chavarria et al. (2014) was used to manufacture negative photo-lithographic chrome masks for fluidic and control channels using maskless lithography. This step was followed by the fabrication of two master moulds using contactlithography. The master moulds were comprised of SU-8 
cured onto silicon substrates. By casting PDMS against the master moulds, the microchannel features on the moulds were replicated on the PDMS slab. Afterwards, the control channel PDMS replica was peeled off its mould and aligned on the fluidic channel PDMS replica and both were then baked overnight. Finally, the complete PDMS slab was peeled off the mould and plasmacleaned with oxygen and bonded irreversibly to a coverslip to form a sealed microfluidic device.

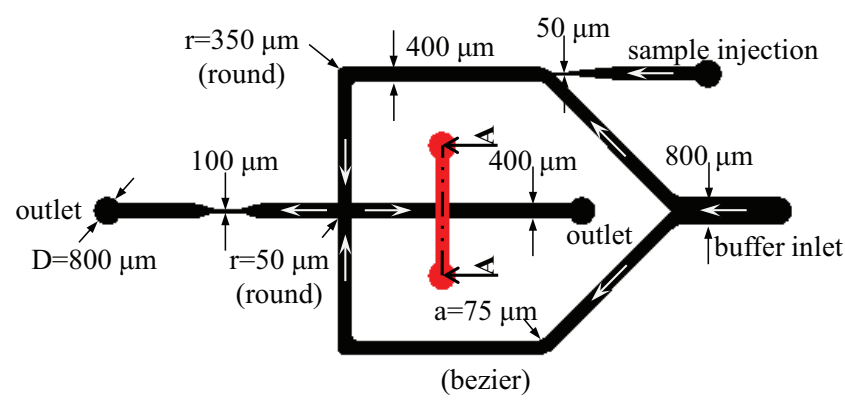

Fig. 2: Cross-slot microchannels fabricated in this work (black: fluidic channel, red: control channel)

\section{Basic concepts of the controlled microfluidic hydrodynamic trap}

Functioning of the microfluidic trap is based on the active flow manipulation of the stagnation point to confine objects at the centre of the cross-junction microchannel. Here, a flow-based technique, introduced by Tanyeri et al. (2010), was employed to confine microorganisms at the stagnation point, thereby exposing them to long-term straining flow. This method is an image-based control technique in which the stagnation point is actively repositioned using an on-chip membrane valve to push a target object towards the centre of the cross-slot channel/centre of the trap. In this study, due to the limited resolution of the camera (necessary to decrease the delay time in real-time control) and the shape of the objects, detecting the connections of the filaments of $A$. circinalis (See ESI section 1 for culture growth and maintenance conditions) is challenging. Therefore, a different image processing method compared to Tanyeri et al. (2010) was used. In Appendix A, we describe the mechanism of the hydrodynamic microfluidic trap including continuous stagnation point repositioning (A.1), automation of the stagnation point repositioning (A.2), feedback control algorithm (A.3) and inline image processing (A.4).

\section{Experimental setup - controlled microfluidic} hydrodynamic trap

A programmable syringe pump (Harvard PHD ULTRA) was used to deliver the buffer and sample fluids (e.g. MLA medium containing A. circinalis) into the microfluidic device through a 1000 and $250 \mu \mathrm{L}$ gas-tight glass syringes (Hamilton), respectively. Bent metal tubing (24 gauge, zephyrtronics) and Luer lock adaptors (Upchurch) were used to establish the connection between the inlets of the microfluidic device and syringes via the PFA tubes (0.020 in ID, $1 / 16$ in OD, IDEX) respectively. A manual micro shut-off valve (1/16 in OD tubing, Upchurch) was used to block the injection port after a target is trapped in the cross-junction. The same metal tubing and PFA tubes were used to establish the connection between the microfluidic device and the drain reservoir and also to establish the connection between the electronic pressure regulator and the control channel (on-chip membrane valve). The outlet tubes were of equal length and both were submerged into a sample container, thereby maintaining a constant pressure drop in the outlets. An essential oil was used to fill the control channel to avoid permeating the Nitrogen to the fluidic channel, which disrupts the water flow and control system.

The microfluidic device was mounted onto an inverted TI-U Eclipse Nikon microscope equipped with a stage-up configuration (two filter block turrets), in which simultaneous imaging with two cameras was possible. This configuration enables streaming the images using a low resolution and relatively high frequency camera to control the flow, and simultaneously acquiring high definition movies using a DSLR camera. For the control, a low-resolution monochrome PointGery camera (Blackfly 0.3 MP, GigE PoE) capable of streaming images at $90 \mathrm{fps}$ at full resolution was coupled to back port of the microscope using a $0.5 \times$ relay lens. For video acquisition, a Nikon D800 camera was used to acquire high resolution (1080p) colour images at 30 fps.

Images were acquired through a $20 \times$ objective lens (CFI S Plan Fluor ELWD). A filter cube holding a $50 / 50,45^{\circ}$ mirror was placed into one of the microscope turrets to split the light between the two cameras. No extra light source was used to illuminate the flow for imaging apart from the ambient room lights.

The microfluidic trap is controlled by a custom-built MATLAB code in which inline image processing and online controlling of the devices are carried out (see Appendix A.4 for the inline image processing). The control camera (PintGrey) that is directly controlled in the MATLAB environment takes an image of the cross-slot 
junction when required and streams it to a computer. To control the electronic pressure regulator and the syringe pump, an Arduino UNO controller board was employed (see Appendix A.3 for the feedback control algorithm). The controller is used to send 0-5 volt digital signals from MATLAB through its pulse-width modulation (PWM) pins. The signal is amplified to a $0-10$ volt signal, suitable for the input of the pressure regulator.

To pressurise the control channel, a 2000 psi Nitrogen tank was used. Using a manual valve the pressure at the outlet of the tank is reduced to $33 \mathrm{psi}$, suitable for the inlet of the electronic pressure regulator. A high resolution electro-pneumatic pressure regulator (Proportion-Air, QPV series) with an accuracy and resolution of $\pm 0.2 \%$ and $\pm 0.005 \%$, respectively, at full scale was calibrated to provide the outlet pressure at 0-30 psi. This covers the range of the required pressure in the trap simulation explained in ESI section 2. However, the pressure regulator is capable of responding for a pressure change of $1 \mathrm{psi}$, within 5 and $11 \mathrm{~ms}$ when pressurising and relieving the pressure, respectively. This leads to an upper limit of roughly $90 \mathrm{~Hz}$ (the same as the camera frequency) for the response time, given that one only needs to change the pressure by 1 psi to keep a particle at the stagnation point. However, according to the simulation of the microfluidic trap (see ESI section 2), after the initial confinement of a target, less than 0.5 psi is sufficient to trap the targets.

\subsection{Experimental procedure}

In contrast with previous studies that either confined micro-sized objects (e.g. Tanyeri et al. 2010) for long time scales (minutes to hours) and low strain rate (about $1 \mathrm{~s}^{-1}$ ) or short time scales (De Loubens et al., 2015) (a few milliseconds) and high strain rate (about $500 \mathrm{~s}^{-1}$ ), here we achieved the confinement of the micron-sized objects at relatively high strain rates (up to $42 \mathrm{~s}^{-1}$ ) for a relatively long time (up to an hour). This was conducted by programming and synchronising the pump with other equipment and gradually increasing the flow rates using different step functions (see Appendix A for the full details of microfluidic operation including feedback control system (A.3), inline image processing (A.4) and calibration of the trap (A.5)).

Prior to operating the control system, the syringe pump delivered the fluid through the buffer inlet port at a high flow rate $(4000-5000 \mu \mathrm{L} / \mathrm{hr})$ for about two minutes. Delivering the fluid to the micro-channels at high flow rates assists in removing bubbles from the channels. The flow rate was gradually decreased to 50 $\mu \mathrm{L} / \mathrm{hr}$. Prior to injecting the targets, the control system was operated to compute the background (cross-region)

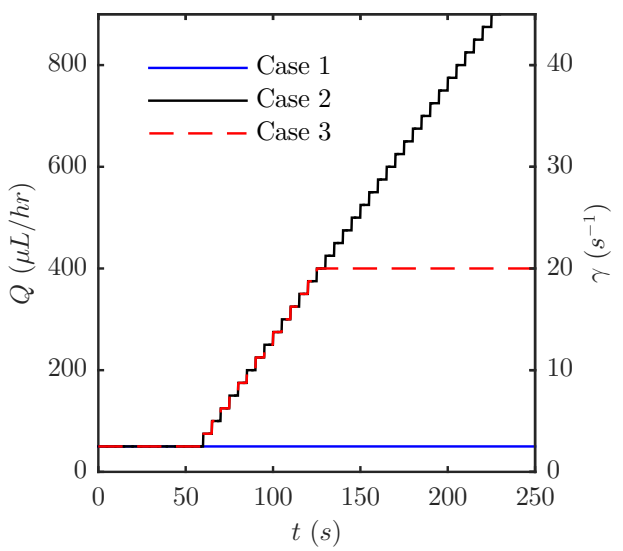

Fig. 3: Flow rate and strain rate variations to be applied in the microfluidic trap after confining a target object.

and then the shut-off valve was manually opened to inject the targets. Once a target was trapped, the valve was closed manually. After about one minute of trapping, the flow rate was either kept unchanged or was increased using the functions (case 1,2 and 3) shown in figure 3 . The right-hand side axis of the figure shows the strain rate of each flow rate obtained from micro-PIV experiments in the cross-slot channel with the absence of target objects (Akbaridoust, 2017; Akbaridoust et al., 2018). The three functions were defined within the pump inbuilt-interface The pump was connected to the Arduino controller, thereby sending signals to MATLAB and recording the exact time of triggering the flow rate functions.

\section{Simultaneous micro-PIV measurement and real-time control in the hydrodynamics trap}

In the microfluidic trap, the necessity of having a relatively large field of view in the control camera (i.e. the whole cross-junction) limits the use of high magnification microscope objective lenses. Therefore, it is not possible to simultaneously control the flow in the cross-slot channel and measure the velocity field with high resolution near the trapped object's body. Moreover, classical micro-PIV measurement techniques are limited to measuring a two-dimensional velocity field. Hence, apart from the PIV results, we adopt the analytical solution of the flow around an ellipsoidal-shaped object in the straining flow to obtain the velocity fields. However, it should be noted that one cannot solely rely on the analytical solution of the straining flow over an object, as analytical solution requires the strain rate of the undisturbed flow (away form the trapped object). Although, in previous works, researchers assumed that when the objects are trapped, the strain rate in 


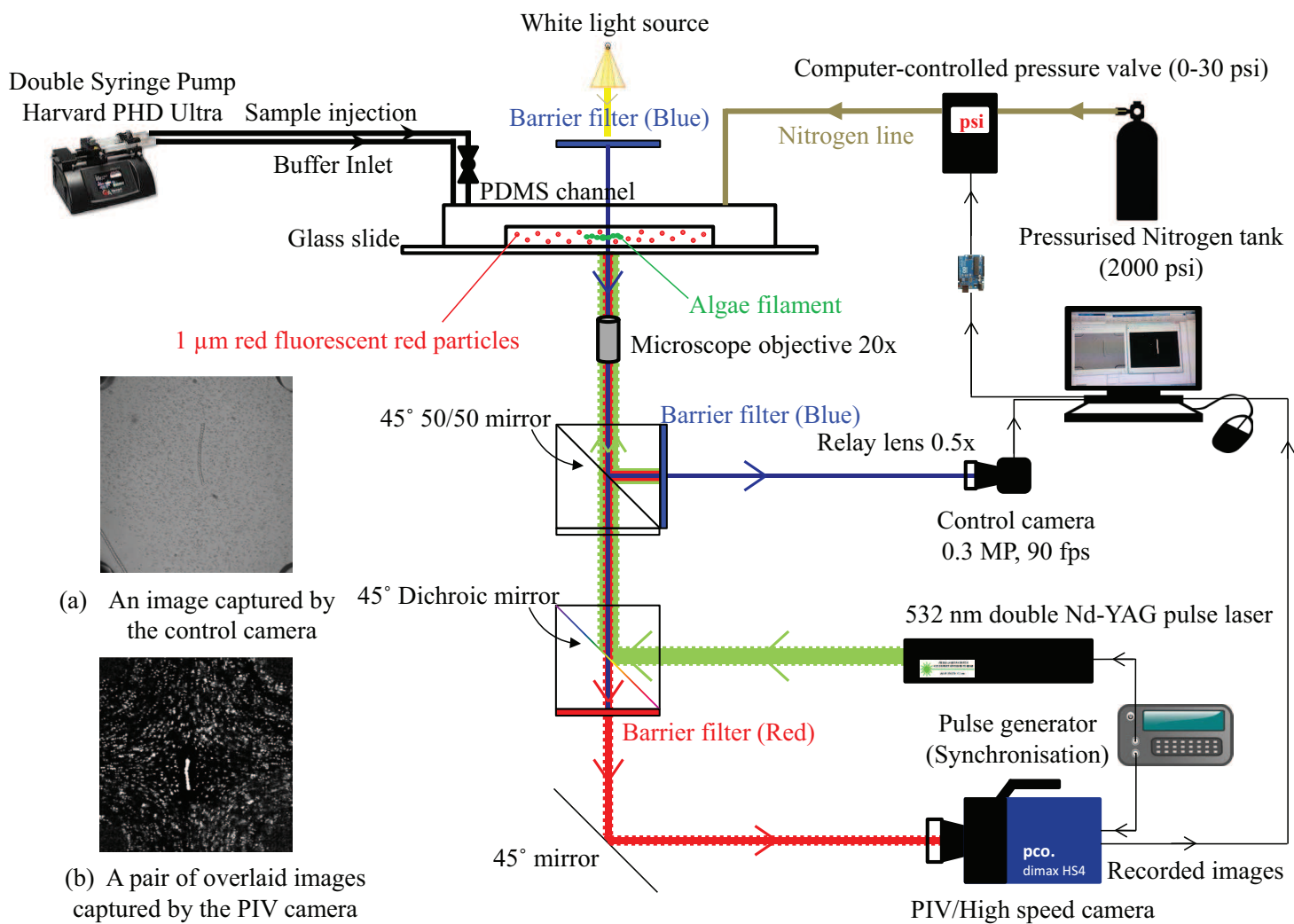

Fig. 4: Schematic of the modified simultaneous micro-PIV and control experimental setups.
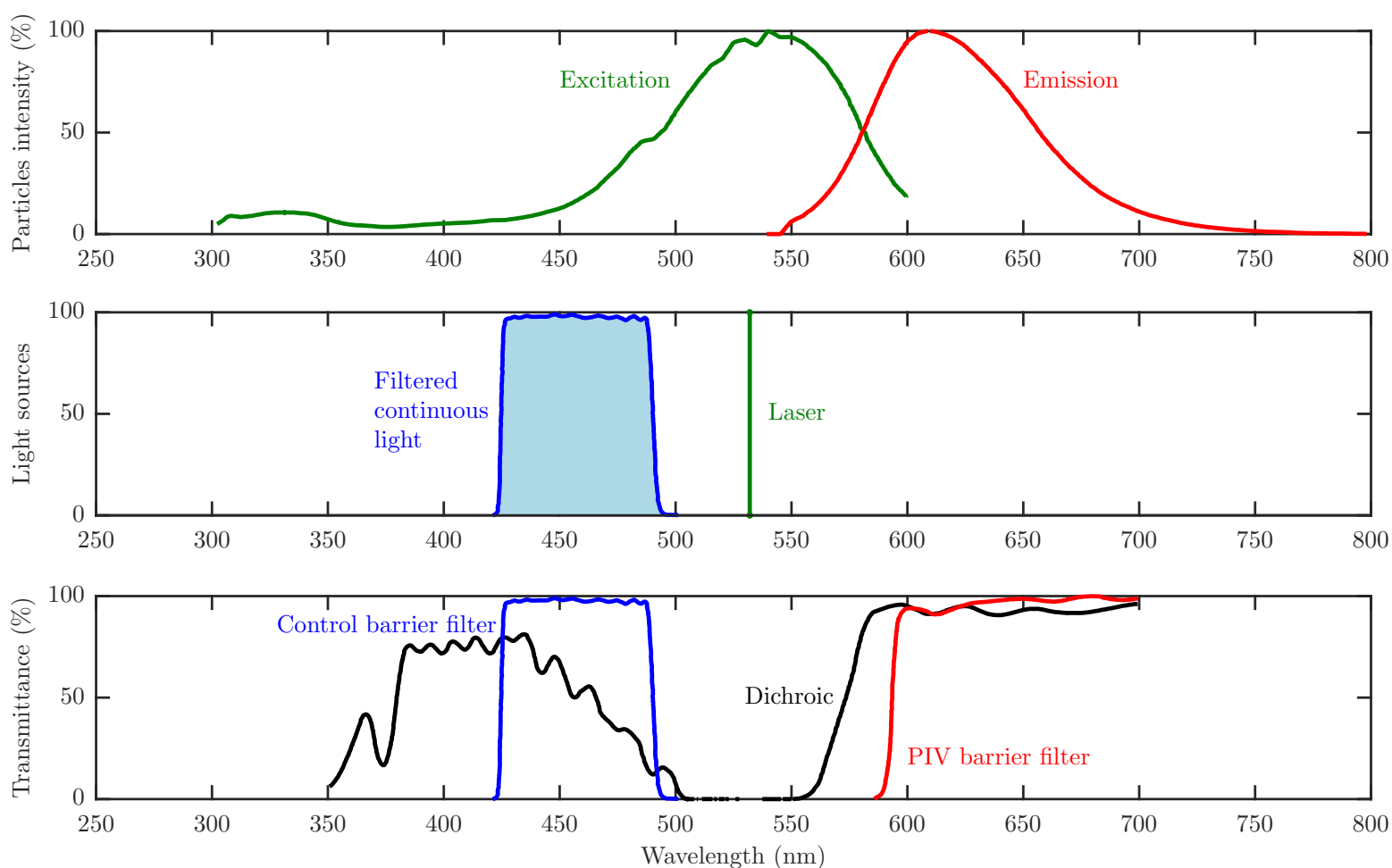

Fig. 5: Excitation and emission of fluorescently labelled particles and transmittance of barrier, single-band bandpass filters and dichroic mirror (the data were received from Thermo Fisher, Nikon Instruments Co. and Semrock Inc.). 


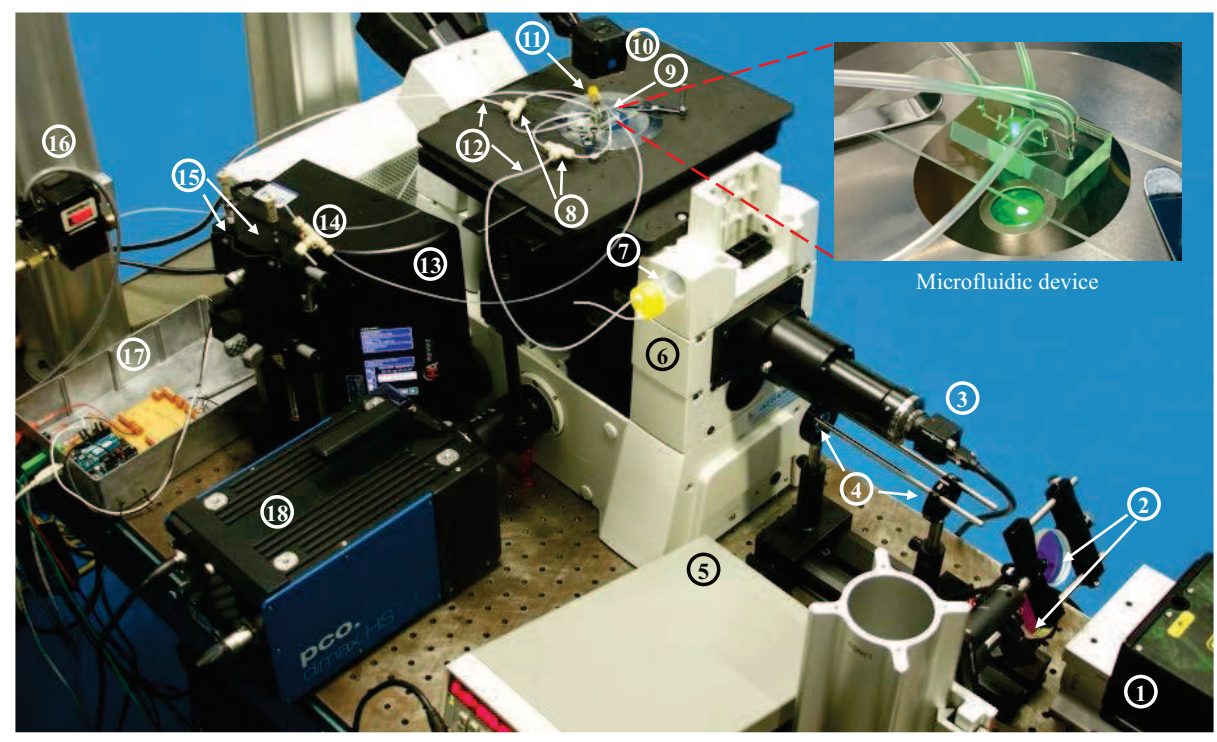

Fig. 6: Photograph of the simultaneous micro-PIV and real-time control experimental setup. 1: Double-pulsed laser, 2: Aligning mirrors, 3: Control camera, 4: Beam expanding lenses, 5: Pulse generator, 6: Inverted microscope, 7: Drained fluid container, 8: T-junction connectors, 9: Microfluidic device, 10: LumeCube light source, 11: Control channel plug, 12: PFA tubing, 13: Double syringe pump, 14: Manual micro shut-off valve, 15: Syringes and connectors, 16: Electronic pressure regulator, 17: Controller and amplifier, 18: PIV camera

the flow is the same as the strain rate of the flow with the absence of the target objects, the trapped object may change the flow (De Loubens et al., 2015; Gossett et al., 2012). In the case of trapping of relatively large objects such as Anabaena circinalis the average flow strain rate (obtained from the experiments in this work) is higher than flow strain rate with the absence of the target (Akbaridoust, 2017; Akbaridoust et al., 2018), due to obstruction from the target object. Hence, using the new micro-PIV measurements we obtain the strain rate with the presence of the target object and substitute it in the analytical solution of the flow over an ellipsoidal object immersed in a creeping straining flow, presented by Jeffery (1922) (see ESI section 3). A combination of the micro-PIV and analytical solution removes the limitation of increasing the resolution of the simultaneous micro-PIV in the controlled straining flow. It should be noted that, one can use 3D Particle tracking Velocimetry (PTV) using a defocused method (Barnkob et al., 2015; Rossi and Kähler, 2014) to avoid using analytical solution, However, the same fundamental optical setup must be used to conduct simultaneous velocity measurement and trapping, and long-term trapping is necessary to acquire enough velocity data points from PTV.

Simultaneous trapping of objects and micro-PIV measurements is challenging because both require their own illumination, optics and camera focused on the mi- cron sized objects. Employment of a specific wavelength separation enabled us to combine a classical micro-PIV and microfluidic trap setups. The limitations of a simplistic combination of these two setups have been detailed in Appendix B.

\subsection{Experiments}

All the components in the microfluidic trap experimental setup explained above and a micro-PIV setup (Akbaridoust et al., 2018) were used except for a few minor changes. As can be seen in figure 4, a filter cube including the $45^{\circ}, 50 / 50$ mirror (that splits light to the control camera and PIV camera) and a single-band bandpass filter (420-480 nm, Semrock) was placed into the upper turret of the microscope. While, the epifluorescent cube (G-2A) filter was placed into the bottom turret of the microscope. Figure 5 shows the diagram of the fluorescent intensity of the particles and the transmittance of the filters that is used to simultaneously conduct micro-PIV measurement and real-time control experiment in the microfluidic trap. Using the specifications of these filters, mirrors and particles enables one to conduct simultaneous micro-PIV and trapping. The DSLR camera in the hydrodynamic trap experiments was replaced by a high speed/PIV camera $(2000 \times 2000$ pixels, 12 bits, PCO.dimax HS4), capable of recording images at $2277 \mathrm{fps}$ at full resolution. 
As it is impossible to filter the ambient room lights, in order to filter the light wavelengths of the constant light source, instead of using the ambient room lights, a LumeCube light source (0-1500 Lumens) with the same single-band bandpass filter (420-480 nm, Semrock) used in front of the 50/50 mirror was used to illuminate the flow. Figure 6 shows a photograph of simultaneous micro-PIV and real-time control experimental setup and its components used in this work.

The $1000 \mu \mathrm{L}$ syringe (connected to the buffer inlets) was filled with water and seeding particles (one micron diameter red fluorescent polystyrene aqueous microspheres, ThermoFisher Scientific), and the $250 \mu \mathrm{L}$ syringe (connected to the injection port) was filled with the culture medium. To illuminate the seeding particles for micro-PIV, a $532 \mathrm{~nm}$ Nd:YAG double-pulsed laser (200 mJ per 9 ns pulse, EverGreen - BigSky Laser Series) that is attenuated and able to double-pulse at 15 $\mathrm{Hz}$ was used. The same procedure in trapping was used to run the microfluidic trap and micro-PIV measurements. The pump was operated at a constant rate of 50 $\mu \mathrm{L} / \mathrm{hr}$ (Case 1 in figure 3 ).

\section{Results and discussions}

Figure 7 shows three partially overlaid images of the A. circinalis strain (ACMR01) that entered the crossslot region and eventually was trapped in the crossslot centre (see Movie S1). The trajectory of the filament's centre of mass is superimposed on the images and compared with the estimated trajectory of an object in the straining flow without active control (obtained from micro-PIV results in our previous work (Akbaridoust et al., 2018)). The experimentally measured trajectory of the target is also compared with the simulated trajectory of the target entered at the same position in the same flow rate/strain rate with the same trap centre.

As previously mentioned, with the intention of demonstrating the capability of the microfluidic trap for confinement of both motile and non-motile target objects, we also trapped $D$. tertiolecta species. Figure 8 shows a trapped $D$. tertiolecta cell and its trajectory in the microfluidic device. The experimentally determined trajectory of the $D$. tertiolecta agreed well with the simulation results (shown in figure 8), whereas a discrepancy in the experimental results and numerical results can be seen in the case of the trapped $A$. circinalis (shown in figure 7 ). The discrepancy might be rooted in the fact that in the simulation, the target object is simulated by its centroid (a point moving in the straining flow). However, the relatively large

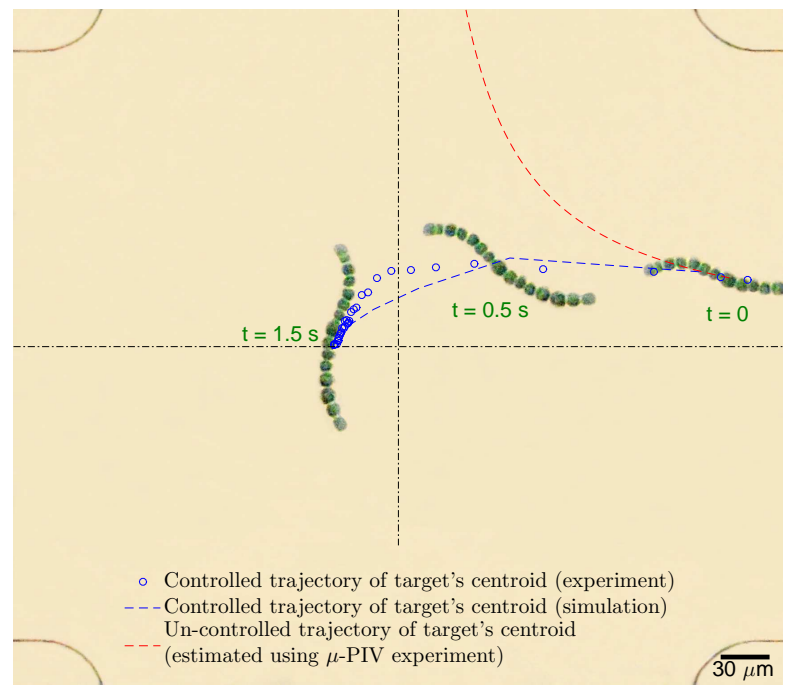

Fig. 7: Three partially overlaid images of the confinement of the $A$. circinalis filament (ACMR01) in the cross-slot junction using active control system at $Q=50 \mu \mathrm{mL} / \mathrm{hr}$ and $\gamma=2.3 \mathrm{~s}^{-1}$ (See Movie S1). The experimentally measured target's trajectory is shown by blue circles, whereas the blue dash line shows the numerically estimated target's trajectory. The red dash line shows the streamline of the flow with the absence of the control system (obtained from micro-PIV experiments).

size of the A. circinalis and its filamentous morphology results in disrupting the hyperbolic streamlines of the flow, whereas the Dunaliella, which is much smaller than $A$. circinalis and spherical in shape, follows the flow streamlines with less influence on them. Therefore, there was less discrepancy between the simulation and the experimental results.

\subsection{Simultaneous micro-PIV results}

Using the simultaneous micro-PIV and trapping method in the cross-slot microchannels, the velocity field around the trapped filaments were measured. Figure 9 shows an example of six overlaid image pairs captured by the PIV camera sensor while a filament of $A$. circinalis was trapped in the cross-junction (see Movie S2). For the purpose of displaying the image, the image was post-processed and converted to a black and white image. Therefore, the particles appear slightly larger than the real size captured by the camera sensor. Apart from the red tracer particles, images of the blue-green $A$. circinalis filament were recorded by the PIV camera sensor, in spite of the presence of the barrier filter (that blocks all the wavelengths except red) with the specifications shown in figure 5 . This is due to 


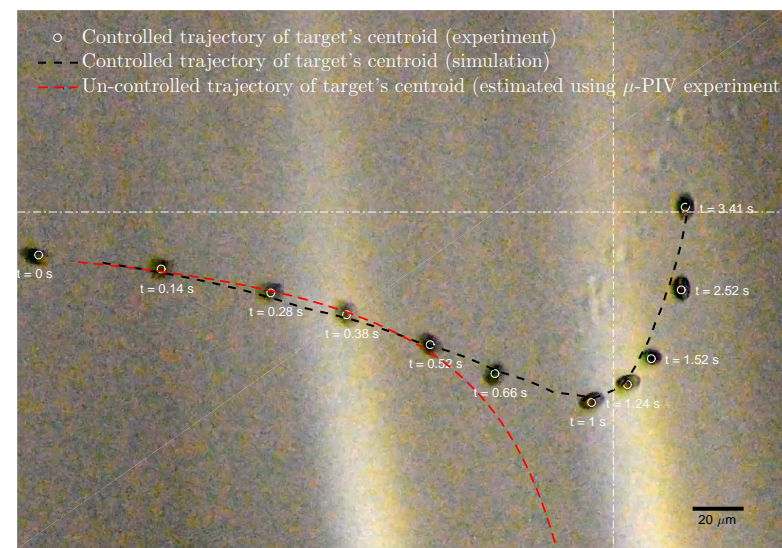

Fig. 8: Eleven partially overlaid images of the confinement of a Dunaliella tertiolecta in the cross-slot junction using an active control system at $Q=100 \mu \mathrm{mL} / \mathrm{hr}$. The experimentally measured target's trajectory is shown by white circles, whereas the black dash line shows the numerically estimated target trajectory. The red dash line shows the streamline of the flow with the absence of the control system.

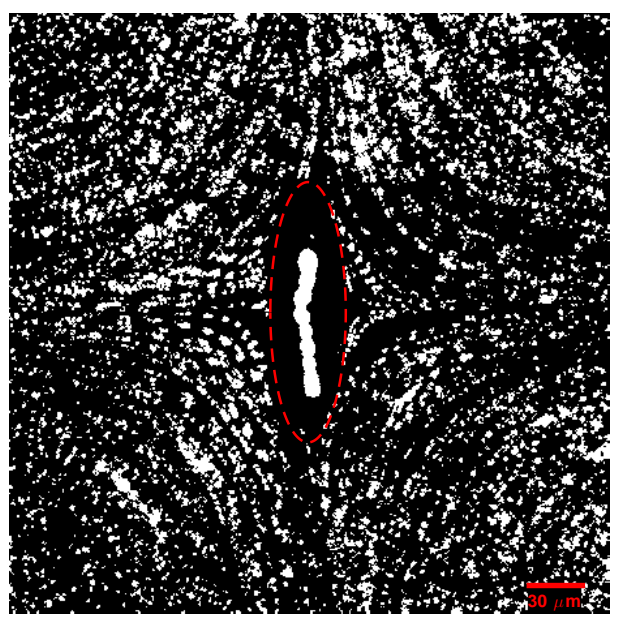

Fig. 9: An example of six overlaid image pairs while a filament of $A$. circinalis was trapped in the cross junction (see Movie S2).

the existence of the chlorophyll in the A. circinalis filament. Although chlorophyll appears green when it absorbs white light, regardless of the light source, it emits red wavelength as well (Hall et al., 1993). In the figure it is noted that the filament is surrounded by a no particle/flow region (shown with the red dash line in figure 9) indicating the presence of mucilage around the filament. Cyanobacteria produce mucilaginous sheaths to protect themselves from other deleterious microorganisms (Amsler, 2008; Pajdak-Stós et al., 2001). As the mucilage was not washed off from the filament while exposed to the straining flow, we consider both the filament and the sheath a single solid object.

The same pre-processing, processing and postprocessing techniques used in our previous work (Akbaridoust, 2017; Akbaridoust et al., 2018) were implemented to calculate the velocity field in the cross-slot junction of the microchannel.

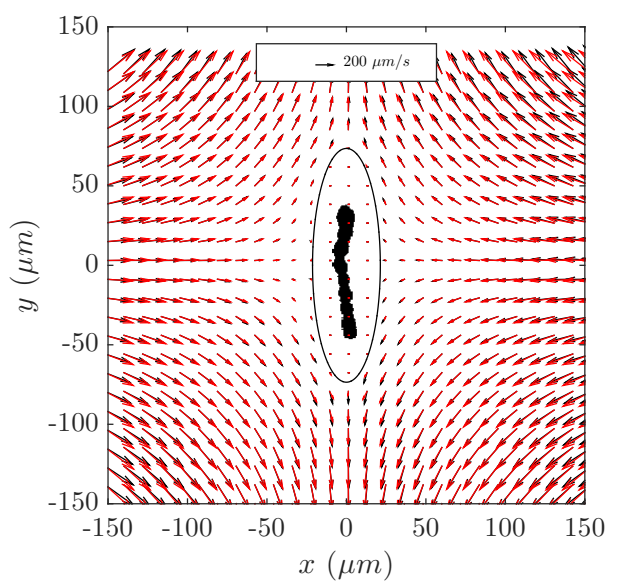

Fig. 10: Comparison of the ensemble-average velocity vectors (black vectors) obtained from micro-PIV measurement around a filament of the A. circinalis (shown in figure 9) and the velocity vectors (red vectors) analytically obtained from Jeffery (1922) around an ellipsoid.

To find the strain rate $(\gamma)$, which is required to be substituted in the analytical solution (see ESI section 2.2 ), the gradients of the velocity field away from the trapped object is calculated using a least square fitting method used in our previous work (Akbaridoust et al., 2018). The calculated strain rate was $\gamma=3.2 \mathrm{~s}^{-1}$ at $Q=50 \mu \mathrm{L} / \mathrm{hr}$, whereas $\gamma=2.3 \mathrm{~s}^{-1}$ at the same flow rate with the absence of the trapped filament (Akbaridoust et al., 2018) results in a change of about $40 \%$. By substituting this value into the analytical solution, the velocity field obtained from micro-PIV and analytical solution are compared in figure 10. The black vectors in figure 10 are the ensemble-average velocity vector map in the cross junction around the trapped filament shown in figure 9, while the red vectors that match closely with the micro-PIV results show the analytical solution by Jeffery (1922).

In order to more clearly show the agreement between the PIV results and analytical solution, the $u$ component velocity profile at $y=0$ (along the compressional axis) is plotted in figure 11 and also compared with the velocity profile when the flow was not dis- 


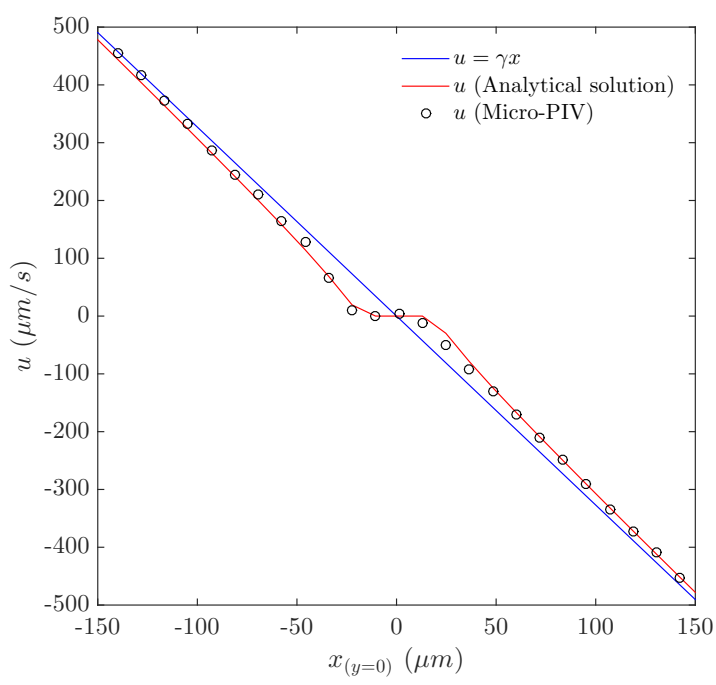

Fig. 11: Comparison of the $u$-component velocity profile at $y=0$ (along the compressional axis) obtained from micro-PIV measurements and analytical solution (Jeffery, 1922) and pure straining flow.

turbed by the trapped filament. As can be seen, the gradient of the measured velocity field is higher by about $40 \%$ than that of the velocity with the absence of the trapped filament $(u=\gamma x)$. This is due to the crossregion being occupied by the trapped target, which in turn reduces the cross-slot area, increases the flow speed and hence the strain rate.

In addition to the strain rate of the flow far from the object with about $40 \%$ deviation, the strain rate in the whole cross-slot region was deviated from the pure straining flow. Figure 12 shows the contour of the strain rate around the object calculated from microPIV measurements (figure 12a) and analytical solution (figure 12b). The slight asymmetric contour map in figure $12 \mathrm{a}$ is due to the asymmetric shape of the filament and the sheath that it is encapsulated with. The strain rate contour reveal that the ellipsoidal object experienced a higher extensional force than compressional force, where the strain rate in the extensional region is about three times higher than that of the pure straining flow and in the compressional region is half of strain rate in pure straining flow.

In our experiments we observe neither mechanical damage to, nor any dramatic deformation in, the filaments of cyanobacteria. Therefore, at present it is challenging to obtain the mechanical properties of the cyanobacteria from the experiments. However, one can calculate the mechanical properties of an ellipsoidal object using the present experimental setup and measurement techniques if the object is deformed.

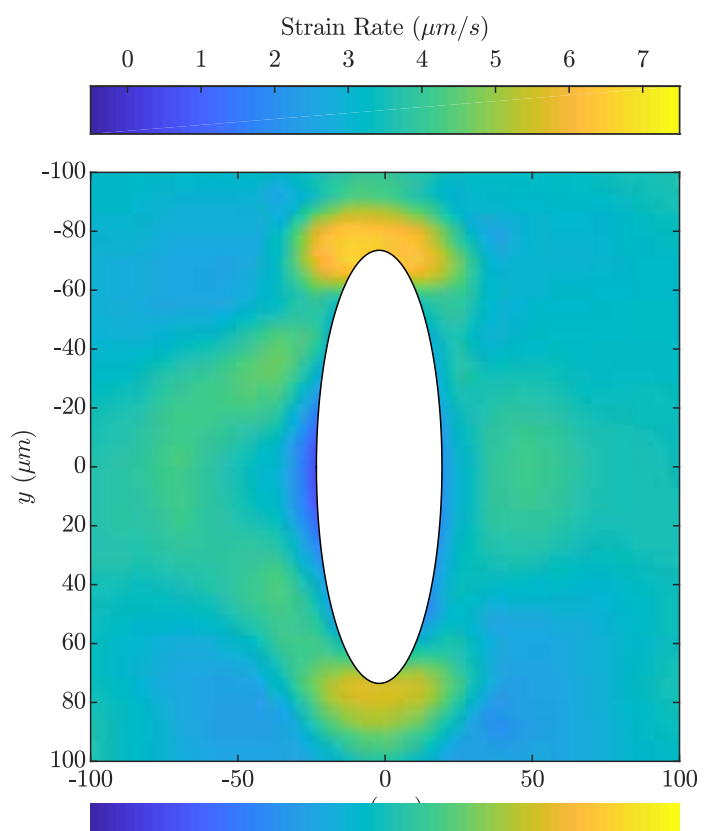

(a) micro-PIV

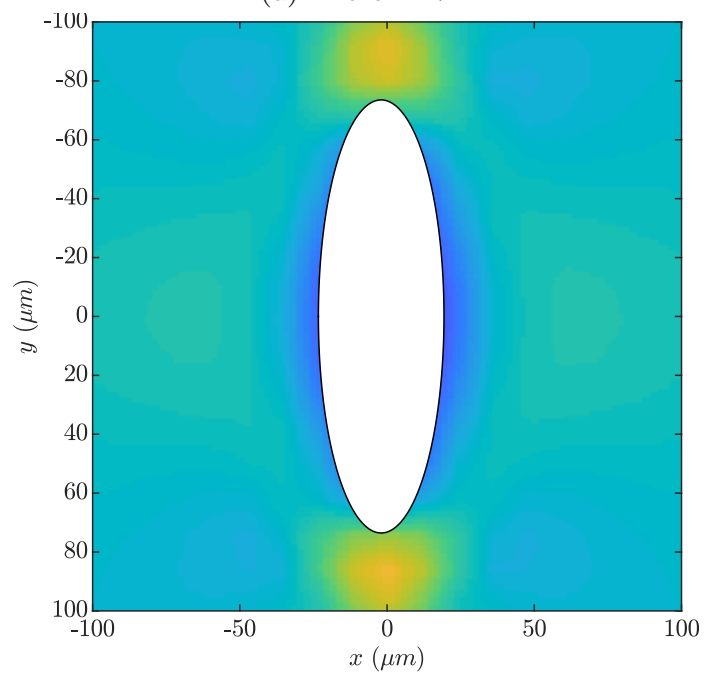

(b) Analytical solution

Fig. 12: Spatial variation of the strain rate in the crossslot region around the trapped target.

\section{Summary and conclusions}

In this paper we reported the use of a fully quantified stagnation-point flow in a cross-slot type microfluidic device equipped with an image-based real-time control system and simultaneously quantified by microPIV measurements.

In the first set of experiments, the microorganisms were confined in the cross-slot type microfluidic device using the image-based active control system. Using a specific combination of image processing techniques resulted in accurate detection and confinement of the fil- 
aments of $A$. circinalis. Due to the small size of the cell connections in $A$. circinalis filaments and using a camera with a low resolution (necessary to operate the active control system at a high speed), the cell connections were not detectable by the control camera. Using a morphologically close image-processing operation by a rectangular-shaped structural element, we increased the connectivity of the blobs without distorting them, thereby computing the correct position of the centroid of the target.

A large number of round motile (Dunaliella tertiolecta) and filamentous (A. circinalis) non-motile microorganisms were successfully trapped for up to an hour at the stagnation point of the controlled straining flow. Prior to the experiments we predicted the trajectory of the target object in the controlled flow. The experimentally determined trajectory of the D. tertiolecta agreed well with the simulation results, whereas for $A$. circinalis a discrepancy in the experimental results and numerical results was observed. The discrepancy is likely due to the fact that in the simulation, the target object is simulated by its centroid (a point moving in the straining flow). However, the relatively large size of the $A$. circinalis and its filamentous morphology results in disrupting the hyperbolic streamlines of the flow, whereas the $D$. tertiolecta, which is much smaller than $A$. circinalis and spherical in shape, follows the flow streamlines with less influence on them.

In contrast with previous studies that either confined micro-sized objects for long time scales (minutes to hours) and low strain rate (about $1 \mathrm{~s}^{-1}$ ) (Johnson-Chavarria et al., 2011, 2014; Latinwo et al., 2014; Li et al., 2015; Shenoy et al., 2015; Tanyeri and Schroeder; Tanyeri et al., 2010, 2011) or short time scales (a few milliseconds) and high strain rate (about $500 \mathrm{~s}^{-1}$ ) (Cha et al., 2012; Gossett et al., 2012; Henon et al., 2014; Henry et al., 2013), we achieved the confinement of the micron-sized objects at relatively high strain rates (up to $42 \mathrm{~s}^{-1}$ ) for a relatively long time (up to an hour). This was conducted by programming and synchronising the pump with other equipment and gradually increasing the flow rates using different step functions. This enables one to use this platform to study the effect of high and long-term straining flow on single micron-sized objects.

In a second set of the experiments, micro-PIV measurements and real-time control were simultaneously carried out in order to measure the velocity field around a trapped target. Two major problems were encountered by combining the two experimental setups (microPIV setup and microfluidic trap setup). The first issue was the disruption of the control camera by the laser pulses that prevents the detection of a target object in the micro-cross channel. The second issue was the production of extremely bright background in the images acquired by the PIV camera sensor due to the constant white light source, which precludes correlating the recorded images.

We successfully implemented a modification to the experimental setup to address the two issues explained above. To tackle the first issue, we replaced the continuous white light with a continuous blue light source (i.e. filtering all the wavelengths except blue). Whilst, to address the second issue, we filtered all the wavelengths (except blue) incident on the control camera sensor. Using this arrangement, the control camera sensor does not capture the laser pulses, and the PIV camera does not acquire the bright background caused by the constant light source.

Carrying out these experiments and combining the experimental results with an analytical solution of flow around an ellipsoidal object immersed in a straining flow (Jeffery, 1922) enables one to accurately obtain the velocity near the trapped target, regardless of the micro-PIV resolution.

In the case of trapping relatively large objects such as $A$. circinalis, the average flow strain rate (obtained from the experiments) was higher than flow strain rate with the absence of the target, due to obstruction from the target object. The increase in the strain rate was about $40 \%$. However, this depends on the size of the object compared to the dimensions of the cross-slot junction.

Acknowledgements The authors gratefully acknowledge the Australian Research Council for the financial support of this work. This work was performed in part at the Melbourne Centre for Nanofabrication (MCN) in the Victorian Node of the Australian National Fabrication Facility (ANFF).

\section{Appendix A: Mechanism of the microfluidic hy- drodynamic trap}

\section{A.1 Continuous stagnation point repositioning}

If the two outlets of the cross-channel have the same flow resistance, the inlet streams are equally bifurcated to the two outlets. If there is a constraint on one of the outlets (e.g. the constraint shown at the bottom outlet in figure A.1a), the outlet flow rates will no longer be the same because of the inequality in the flow resistance of the outlets. Moreover, the stagnation point is displaced towards the outlet with higher flow resistance. Therefore, if the cross-section area of the outlets constantly varies, the stagnation point will be continuously repositioned along the extensional axis.

In this work, repositioning the stagnation point (shown in figure A.1b) is conducted by implementing a fixed width constriction in one of the outlets, and a variable height constriction on the other (Tanyeri et al., 2010). The former is 


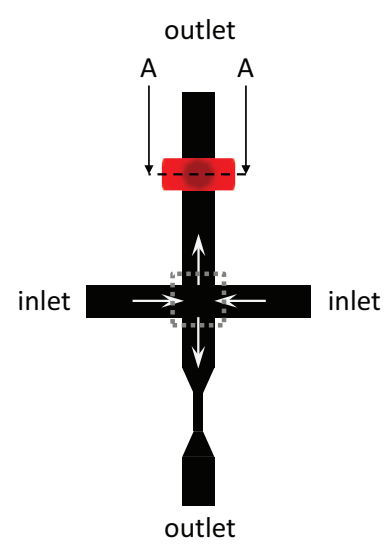

(a) The cross-slot junction with a fixed width constriction (bottom outlet) and a variable height constriction (the red feature on the top outlet).

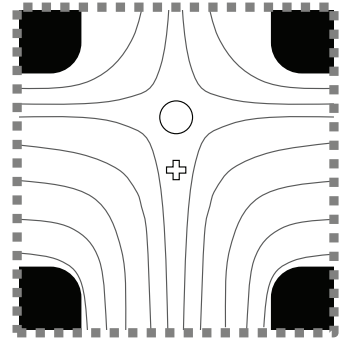

(b) The repositioned stagnation point (shown with a circle), moved towards the top outlet in figure A.1a.

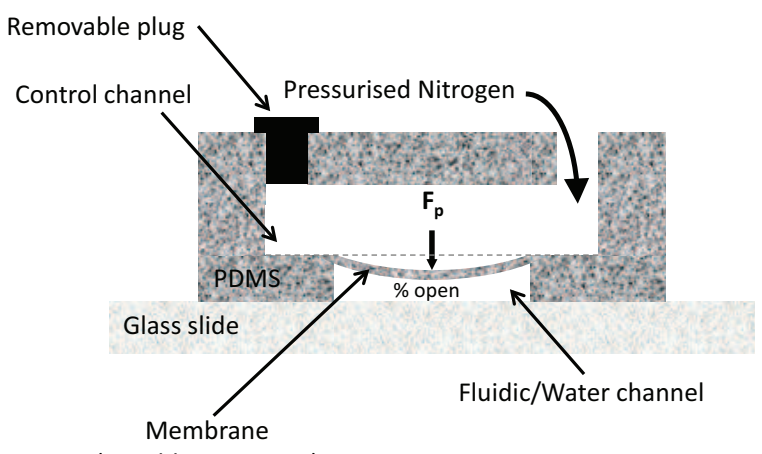

(variable constraint)

(c) The side A-A section of the channel in figure A.1a and the deflected membrane valve that constrained the height of the channel.

Fig. A.1: Different views of the cross-slot junction with the two fixed and variable constraints on the outlets and the effect of the constraint on the stagnation point position and streamlines.

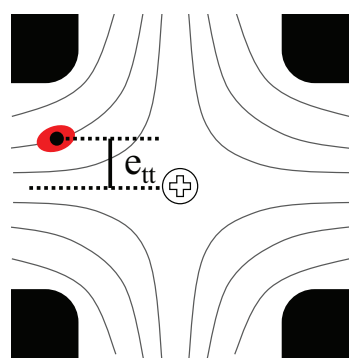

(a) An object (shown by the red ellipse) entering the cross-slot region. The control camera streams the image of the crossregion to a computer to detect the object and find its centroid.

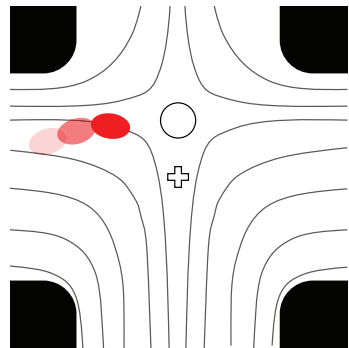

(b) Once the centroid of the object in figure A.2a is determined, the control layer is pressurised and the stagnation point and streamlines are displaced, this resulted in forcing the object to move towards channel centre.

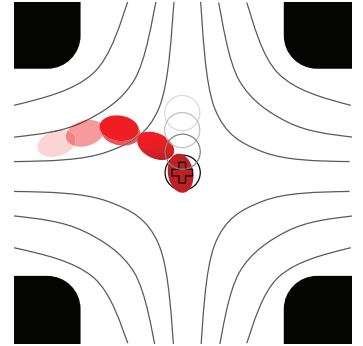

(c) After the target was forced to move towards the centre of the crossjunction, by changing the pressure in the control channel the stagnation point moves towards the centre, too. Eventually, both the stagnation point and the target converged at the centre.

Fig. A.2: The effect of displacing the stagnation point on the flow streamlines and the trajectory of an object in cross-junction. The red ellipse, solid black circle, hollow circle and the hollow cross represent the target object, its centroid, the stagnation point and the centre of the channel, respectively.

shown in the bottom outlet and the latter is shown in red on the top outlet in figure A.1a. Figure A.1c shows the section A-A of the variable height constriction, known as an on-chip membrane valve (in figure A.1a), and how its deformation constricts the height of the fluidic channel. The control channel is filled with pressurised gas, which results in the deflection of the thin membrane, thereby constricting the height of the fluidic channel. It is called the variable constriction because changes in the gas pressure leads to changes in the deflection of the membrane, and consequently constrict the channel at different heights.

\section{A.2 Automation of the stagnation point repositioning} to confine a target object at the channel centre

In the microfluidic hydrodynamic trap, when a target object enters the cross-slot region (shown in figure A.2a), a camera captures and streams the image of the cross-junction to a computer. Using image processing methods the shape of the object is determined and the position of the object centroid is computed. Based on the location of the object in extensional direction, the ratio of the outlet flow rates is changed and consequently the stagnation point is repositioned. The repo- 
sitioning is carried out using the on-chip membrane valve, which allows the flow streamlines to be manipulated. This manipulation places the target object between the stagnation point and the centre of the channel (shown in figure A.2b), thereby exerting a hydrodynamic force on the target object towards the trap centre. The manipulation results in placing the object on a new streamline and moving the object towards the channel centre. All of these steps are repeated until both the target and the stagnation point converge in the centre of the trap/channel (shown in figure A.2c), and at this stage the pressure stays unchanged. However, if due to an external or internal disturbance the target object is displaced from the centre, the same procedure is re-applied forcing the object to return to the centre.

\section{A.3 Feedback Control algorithm}

A linear feedback control algorithm was implemented to update the pressure in the control layer and displace the stagnation point using (Tanyeri et al., 2010):

$P_{v a l}=P_{t c}+K_{P} K_{C} e_{t t}$,

where $P_{v a l}$ is the pressure in the control channel (on-chip valve), $P_{t c}$ is the required pressure to keep the stagnation point at the trap centre. The proportional gain is presented by $K_{P}$ and it can be considered as a constant $\left(K_{P}=-1.5\right)$ and $K_{C}$ is a factor to convert pressure to distance. If there is a linear relation between the pressure and the stagnation point position, $K_{C}$ will be a constant. The offset error $\left(e_{t t}\right)$ in equation A.1 is defined as:

$e_{t t}=Y_{t p}-Y_{t c}$

which is the distance between the position of target $\left(Y_{t p}\right)$ and trap centre $\left(Y_{t c}\right)$ along the extensional axis (shown in figure A.2a). Figure A.3 depicts the flowchart of the steps employed for confining a target object in the centre of the cross-slot junction.

\section{A.4 Inline image processing}

In this work, the foreground detection was carried out using the "Computer Vision System" toolbox in MATLAB. This includes acquiring the cross-region as the background and comparing it with the image of the target in the cross-region. Image comparison was followed by determining whether each pixel belongs to the background (channel) or the foreground (target). Before and after the foreground detection, image contrast enhancement and some morphological operations were carried out to improve in locating the accuracy the target's centroid.

Figure A.4 depicts the implementation of the image processing steps used in the present work to locate the centroid of the A. circinalis filament. Firstly, the background image of the region of interest (ROI) was acquired. This was conducted prior to the filament entering ROI (partially shown in figure A.4a). Figure A.4b shows a filament of the cyanobacteria in the ROI. Upon acquiring the image of the target (filament) the image contrast was enhanced to increase pixel intensity of the filament (shown in figure A.4c). Afterwards, the foreground was detected. Foreground detection requires defining

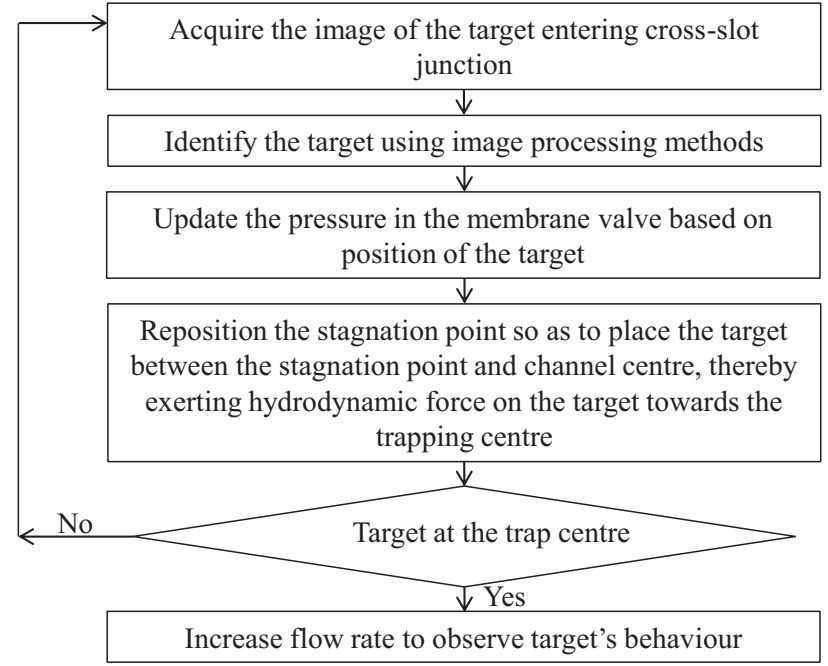

Fig. A.3: Algorithm for confining a target object in the cross-slot region of the microchannel, which is schematically shown in figure A.2.

a threshold parameter that depends on the light condition to determine whether each pixel belongs to the channel or the target. The background was then subtracted from the foreground, thereby achieving the target object mask (the binary image shown in figure A.4d). This step was followed by removing small objects and noises, by defining a certain threshold and removing the blobs (connected white regions) smaller than the threshold (shown in figure A.4e). Due to the low resolution of camera and the shape of the objects, detecting the connections of the filaments is challenging. As can be seen the connections are neither observable in figure A.4b, nor detectable in figure A.4e. To alleviate this issue, we used morphologically close operation by a $7 \times 7$ pixel squareshaped structural element to increase the connectivity of the blobs without over-fattening it. Stated in Smith et al. (1997), morphologically close operation in processing of a binary image includes a morphologically erosive operation (making the blob smaller) followed by a morphologically dilative operation (making the blob larger). Figure A.4f shows the postprocessed mask of the filament after applying the morphologically close operation. Eventually, the centroid of the filament was determined using the blob analysis of the MATLAB computer vision system toolbox.

\section{A.5 Calibrating the microfluidic trap}

The calibration curve of the microfluidic trap is the variation of the stagnation point position at different control channel pressures. The linear region of this curve is used to determine the conversion factor $\left(K_{C}\right.$ in equation A.1). To experimentally determine this curve, a fluid visualisation experiment was conducted, which delivered the fluid seeded with tracer particles to the cross-junction. By capturing the images of the cross-region, the flow is visualised. In this experiment, the flow was illuminated using the Nikon microscope Epifl illuminator (Mercury lamp). The epifluorescence imaging setup configuration that was used in micro-PIV experiments Akbaridoust et al. (2018), except the PIV camera that was 

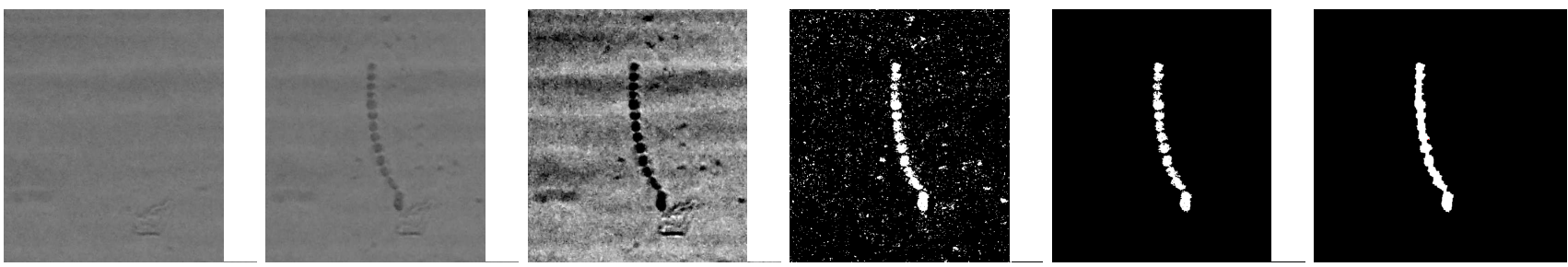

(a) Background image.

of a target object contrast of the orig- foreground.

(filament of $A$. circi- inal image.

nalis). (e) Removing the (f) Improving the small objects and connectivity of the noises form the fore- filament cells using ground. morphologically close operation.

Fig. A.4: Implementation of the image processing steps that were used for foreground detection (detecting the $A$. circinalis filament in the microchannel).

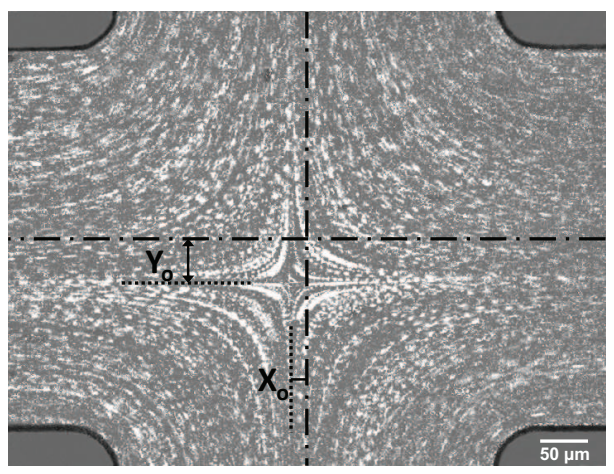

Fig. A.5: An example image of 150 overlaid recordings (at the flow rate of $50 \mu \mathrm{L} / \mathrm{hr}$ and control channel pressure of about $8 \mathrm{psi}$ ) that was used to determine the calibration curve of the microfluidic trap.

replaced by the control camera was employed to acquire the images. The backgrounds (out-of-focus particles) of the acquired images were then removed Akbaridoust et al. (2018) and then 150 images were overlaid. This procedure was repeated at the different pressures in the control channel, and for each set of images the stagnation point was determined manually/visually with the accuracy of one pixel. One pixel corresponds to one micron for the control camera used in this experiment.

Figure A.5 shows an example of 150 overlaid images at the flow rate of $50 \mu \mathrm{L} / \mathrm{hr}$ and control channel pressure of about 8 psi. The distance of the stagnation point position from the centre line of the channel along the extensional and compressional axes are represented by $Y_{o}$ and $X_{o}$, respectively. As can be seen the stagnation point is not located on the vertical centre line, due to the flow added to one of the inlets where the sample injection port is located. However, $X_{o} \neq 0$ only occurs when the injection port is open. In trapping experiments once a target is trapped, the injection port is manually closed and the stagnation point gradually moves on the centre line along the compressional axis. Figure A.6 shows the calibration curve that was experimentally determined (at $50 \mu \mathrm{L} / \mathrm{hr}$ ) at different pressures in the control channel. The slope of the fitted trend line represents the conversion factor $\left(K_{C}\right)$.

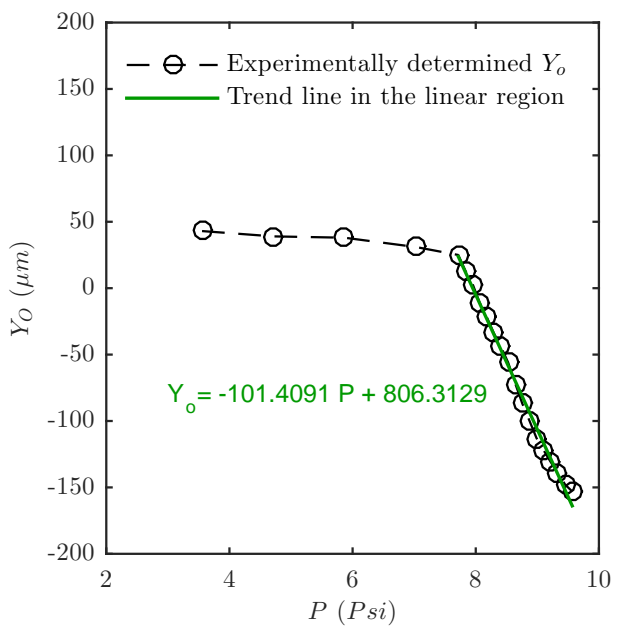

Fig. A.6: Experimentally determined calibration curve of the microfluidic trap. The green line is the trend line fitted to the linear region, and the slope of this line represents the conversion factor $\left(K_{C}\right)$.

\section{Appendix B: Methodology of simultaneous micro-PIV measurements and trapping}

In principal the use of volume illumination in PIV measurements results in bright background in the images that drastically reduce the signal-to-noise ratio and the correlation peak detectability. Therefore, in micro-PIV the epifluorescence imaging technique (i.e. illuminating the fluorescent particles by green pulsed-light and capturing the red light from them) is implemented to alleviate this issue (Santiago et al., 1998). In hydrodynamic microfluidic trap experiments (in this work and also Schroeder and co-workers (Johnson-Chavarria et al., 2011, 2014; Latinwo et al., 2014; Li et al., 2015; Shenoy et al., 2015; Tanyeri and Schroeder; Tanyeri et al., 2010, 2011) a constant white light source (here ambient room lights) was used for imaging. Hence, simultaneous trapping of objects and micro-PIV measurements is challenging because both require their own illumination, optics and camera focused on the micron sized objects. Simply combining the two experimental setups (micro-PIV and the microfluidic trap setups) to conduct simultaneous micro-PIV 


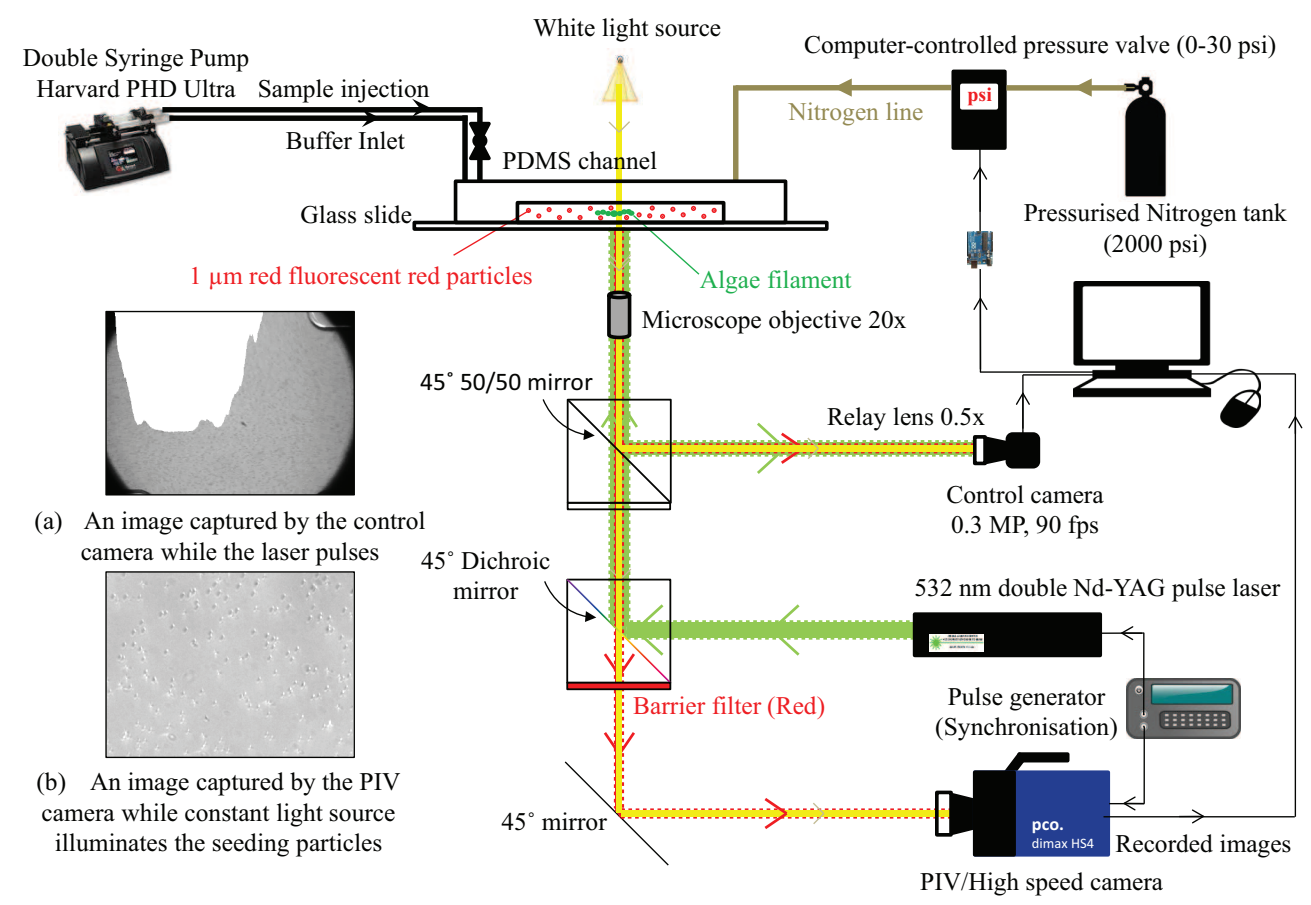

Fig. B.1: Schematic of the combination of micro-PIV and active control setups and the resulting imaging problems in the images acquired by the PIV and the control camera.

measurements and trapping in the microfluidic device leads to two major problems. The combination of the two setups is shown in figure B.1. The first issue is the disruption of the control camera by the laser pulses. The second issue is the production of an extremely bright background in the images acquired by the PIV camera sensor due to the constant white light source. The former results in preventing the detection of a target object in the cross-slot channel. The top inset image in figure B.1 (figure B.1a) shows an example of the image captured by the control camera when the laser was pulsing. As can be seen, the laser pulse interferes with the image processing techniques for object detection. While, the latter is the production of extremely bright background that precludes correlating the recorded images. The bottom inset image in figure B.1 (figure B.1b) shows an image of the particles captured by the PIV camera, while the constant white light was illuminating the flow.

To tackle the first issue we replaced the continuous white light with a continuous blue light source (i.e. filtering all the wavelengths except blue). Whilst, to address the second issue, we filtered all the wavelengths (except blue) incident on the control camera sensor. Using this arrangement, the control camera sensor does not capture the laser pulses, and the PIV camera does not acquire the bright background caused by the constant light source. Figure 4 shows the schematic of the combined micro-PIV and active control system setups, where the two problems that were pointed out are solved using two single-band (blue) bandpass filters. The bandpass filters block all the wavelengths out of the range of 420-480 nm.

\section{References}

Farzan Akbaridoust. Characterisation of a microfluidic hydro-trap to study the effect of straining flow on wa- terborne microorganisms. PhD thesis, University of Melbourne, 2017.

Farzan Akbaridoust, Jimmy Philip, and Ivan Marusic. A miniature high strain rate device. Proc. 20th AFMC. Conf., 2016.

Farzan Akbaridoust, Jimmy Philip, and Ivan Marusic. Assessment of a miniature four-roll mill and a cross-slot microchannel for high-strain-rate stagnation point flows. Meas. Sci. Technol., 29(4):045302, 2018.

Toh GG Alicia, Chun Yang, Zhiping Wang, and Nam-Trung Nguyen. Combinational concentration gradient confinement through stagnation flow. Lab Chip, 16(2):368-376, 2016.

Charles D Amsler. Algal chemical ecology, volume 468. Springer, 2008.

Arthur Ashkin, James M Dziedzic, JE Bjorkholm, and Steven Chu. Observation of a single-beam gradient force optical trap for dielectric particles. Opt. Lett., 11(5):288-290, 1986.

Rune Barnkob, Christian J Kähler, and Massimiliano Rossi. General defocusing particle tracking. LabChip, 15(17): 3556-3560, 2015.

AL Bernassau, P Glynne-Jones, F Gesellchen, M Riehle, $\mathrm{M}$ Hill, and DRS Cumming. Controlling acoustic streaming in an ultrasonic heptagonal tweezers with application to cell manipulation. Ultrasonics, 54(1):268-274, 2014.

Sukgyun Cha, Taeho Shin, Sung Sik Lee, Wooyoung Shim, Gwang Lee, Seong Jae Lee, Younghun Kim, and Ju Min Kim. Cell stretching measurement utilizing viscoelastic particle focusing. Anal. Chem., 84(23):10471-10477, 2012.

Perran LM Cook, Daryl P Holland, and Andrew R Longmore. Effect of a flood event on the dynamics of phytoplankton and biogeochemistry in a large temperate Australian lagoon. Limnol. Oceanogr., 55(3):1123-1133, 2010. 
Michael D Curtis, Gregory J Sheard, and Andreas Fouras. Feedback control system simulator for the control of biological cells in microfluidic cross slots and integrated microfluidic systems. Lab Chip, 11(14):2343-2351, 2011.

Clement De Loubens, Julien Deschamps, Gwenn Boedec, and Marc Leonetti. Stretching of capsules in an elongation flow, a route to constitutive law. J. Fluid Mech., 767:R3, 2015.

Rebecca Dylla-Spears, Jacqueline E Townsend, Linda JenJacobson, Lydia L Sohn, and Susan J Muller. Singlemolecule sequence detection via microfluidic planar extensional flow at a stagnation point. Lab Chip, 10(12):15431549, 2010.

Charlie Gosse and Vincent Croquette. Magnetic tweezers: micromanipulation and force measurement at the molecular level. Biophys. J., 82(6):3314-3329, 2002.

Daniel R Gossett, TK Henry, Serena A Lee, Yong Ying, Anne G Lindgren, Otto O Yang, Jianyu Rao, Amander T Clark, and Dino Di Carlo. Hydrodynamic stretching of single cells for large population mechanical phenotyping. PNAS, 109(20):7630-7635, 2012.

David G Grier. A revolution in optical manipulation. Nature, 424(6950):810-816, 2003.

David Oakley Hall, JMO Scurlock, HR Bolhar-Nordenkampf, Richard C Leegood, and SP Long. Photosynthesis and production in a changing environment: a field and laboratory manual. Chapman \& Hall London, 1993.

Yann Henon, Gregory J Sheard, and Andreas Fouras. Erythrocyte deformation in a microfluidic cross-slot channel. RSC Advances, 4(68):36079-36088, 2014.

TK Henry, Daniel R Gossett, Yo Sup Moon, Mahdokht Masaeli, Marie Sohsman, Yong Ying, Kimberly Mislick, Ryan P Adams, Jianyu Rao, and Dino Di Carlo. Quantitative diagnosis of malignant pleural effusions by single-cell mechanophenotyping. Sci. Transl. Med., 5(212):212ra163212ra163, 2013

HM Hertz. Standing-wave acoustic trap for nonintrusive positioning of microparticles. J. Appl. Phys., 78(8):4845-4849, 1995

George B Jeffery. The motion of ellipsoidal particles immersed in a viscous fluid. 102(715):161-179, 1922.

Eric M Johnson-Chavarria, Melikhan Tanyeri, and Charles M Schroeder. A microfluidic-based hydrodynamic trap for single particles. J. Vis. Exp., (47):e2517-e2517, 2011.

Eric M Johnson-Chavarria, Utsav Agrawal, Melikhan Tanyeri, Thomas E Kuhlman, and Charles M Schroeder. Automated single cell microbioreactor for monitoring intracellular dynamics and cell growth in free solution. Lab Chip, 14(15):2688-2697, 2014.

Folarin Latinwo, Kai-Wen Hsiao, and Charles M Schroeder. Nonequilibrium thermodynamics of dilute polymer solutions in flow. J. Chem. Phys., 141(17):174903, 2014.

$\mathrm{H}$ Lee, AM Purdon, and RM Westervelt. Manipulation of biological cells using a microelectromagnet matrix. Appl. Phys. Lett., 85(6):1063-1065, 2004.

Yanfei Li, Kai-Wen Hsiao, Christopher A Brockman, Daniel Y Yates, Rae M Robertson-Anderson, Julia A Kornfield, Michael J San Francisco, Charles M Schroeder, and Gregory B McKenna. When ends meet: Circular DNA stretches differently in elongational flows. Macromolecules, 48(16):5997-6001, 2015.

Agnieszka Pajdak-Stós, Edyta Fiakowska, and Janusz Fyda. Phormidium autumnale (cyanobacteria) defense against three ciliate grazer species. Aquat. Microb. Ecol., 23(3): 237-244, 2001.

Jai A Pathak and Steven D Hudson. Rheo-optics of equilibrium polymer solutions: Wormlike micelles in elongational flow in a microfluidic cross-slot. Macromolecules, 39(25): 8782-8792, 2006.

Thomas T Perkins, Douglas E Smith, and Steven Chu. Single polymer dynamics in an elongational flow. Science, 276 (5321):2016-2021, 1997.

Yongqiang Qiu, Han Wang, Christine EM Demore, David A Hughes, Peter Glynne-Jones, Sylvia Gebhardt, Aleksandrs Bolhovitins, Romans Poltarjonoks, Kees Weijer, Andreas Schönecker, et al. Acoustic devices for particle and cell manipulation and sensing. Sensors, 14(8):14806-14838, 2014.

Massimiliano Rossi and Christian J Kähler. Optimization of astigmatic particle tracking velocimeters. Exp. fluids, 55 (9):1809, 2014

J. G. Santiago, S. T. Wereley, C. D. Meinhart, D. J. Beebe, and R. J. Adrian. A particle image velocimetry system for microfluidics. Exp. Fluids, 25(4):316-319, 1998.

C. M. Schroeder, H. P. Babcock, E. S. G. Shaqfeh, and S. Chu. Observation of polymer conformation hysteresis in extensional flow. Science, 301(5639):1515-1519, 2003.

Charles M Schroeder, Eric SG Shaqfeh, and Steven Chu. Effect of hydrodynamic interactions on DNA dynamics in extensional flow: Simulation and single molecule experiment. Macromolecules, 37(24):9242-9256, 2004.

Anish Shenoy, Melikhan Tanyeri, and Charles M Schroeder. Characterizing the performance of the hydrodynamic trap using a control-based approach. Microfluid. Nanofluidics, 18(5-6):1055-1066, 2015.

Anish Shenoy, Christopher V Rao, and Charles M Schroeder. Stokes trap for multiplexed particle manipulation and assembly using fluidics. PNAS, 113(15):3976-3981, 2016.

Steven W Smith et al. The scientist and engineer's guide to digital signal processing. California Technical Pub. San Diego, 1997.

Melikhan Tanyeri and Charles M Schroeder. Manipulation and confinement of single particles using fluid flow.

Melikhan Tanyeri, Eric M Johnson-Chavarria, and Charles M Schroeder. Hydrodynamic trap for single particles and cells. Appl. Phys. Lett., 96(22):224101, 2010.

Melikhan Tanyeri, Mikhil Ranka, Natawan Sittipolkul, and Charles M Schroeder. A microfluidic-based hydrodynamic trap: design and implementation. Lab Chip, 11(10):17861794, 2011

Geoffrey I Taylor. The formation of emulsions in definable fields of flow. Proc. R. Soc. Lond. A, 146(858):501-523, 1934.

Camilo Ulloa, Alberto Ahumada, and María Luisa Cordero. Effect of confinement on the deformation of microfluidic drops. Phys. Rev. E, 89(3):033004, 2014.

Pirjo Wacklin, Lucien Hoffmann, Jiří Komárek, et al. Nomenclatural validation of the genetically revised cyanobacterial genus Dolichospermum (Ralfs ex Bornet et Flahault) comb. nova. Fottea, 9(1):59-64, 2009.

Weilin Xu and Susan J Muller. Exploring both sequence detection and restriction endonuclease cleavage kinetics by recognition site via single-molecule microfluidic trapping. Lab Chip, 11(3):435-442, 2011.

Allen HJ Yang, Sean D Moore, Bradley S Schmidt, Matthew Klug, Michal Lipson, and David Erickson. Optical manipulation of nanoparticles and biomolecules in sub-wavelength slot waveguides. Nature, 457(7225):71-75, 2009. 
A novel combination of classical micro-PIV and real-time flow control setups enabled us to measure the velocity field around a target trapped in the extensional flow, which opens up new vistas of characterisation of the mechanical properties of micron-sized objects.

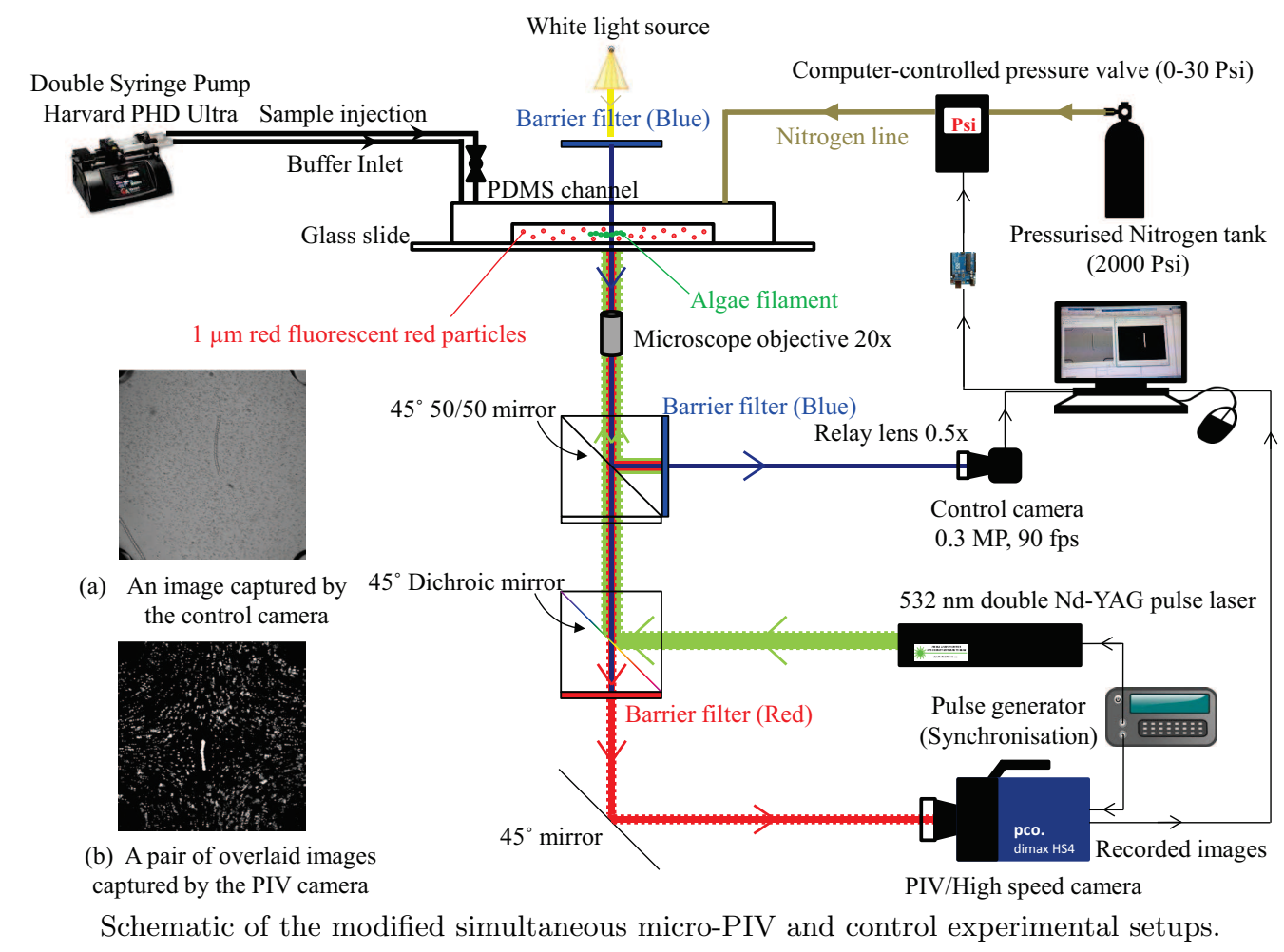




\section{SUPPLEMENTARY MATERIAL \\ Simultaneous micro-PIV measurements and real-time control trapping in a cross-slot channel}

Farzan Akbaridoust ${ }^{a}$, Jimmy Philip ${ }^{a}$, David Robert Allan Hill ${ }^{b}$, and Ivan Marusic ${ }^{a}$

${ }^{a}$ Department of Mechanical Engineering, University of Melbourne, Victoria 3010, Australia

${ }^{b}$ Department of Chemical and Biomolecular Engineering, University of Melbourne, Victoria 3010, Australia

\section{Culture growth and maintenance conditions}

Apart from A. circinalis, we also grew, trapped and exposed the green alga, Dunaliella tertiolecta (the single-celled motile marine species) to demonstrate the capability of the microfluidic trap to confine both motile and non-motile microorganisms and compare the results with the simulation of the microfluidic trap.

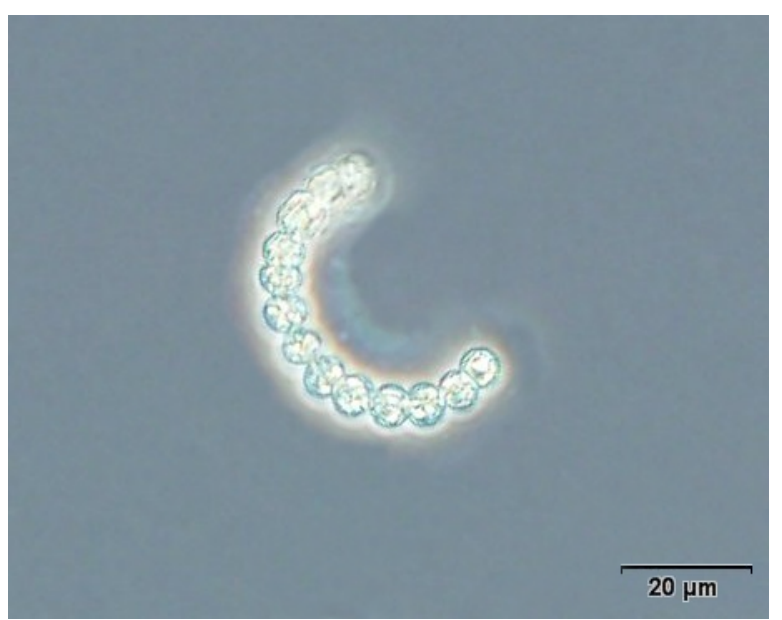

(a) A Anabaena circinalis Rabenhorst strain (CSIRO ID: ACMR01) isolated from Canning River, Perth, Western Australia.

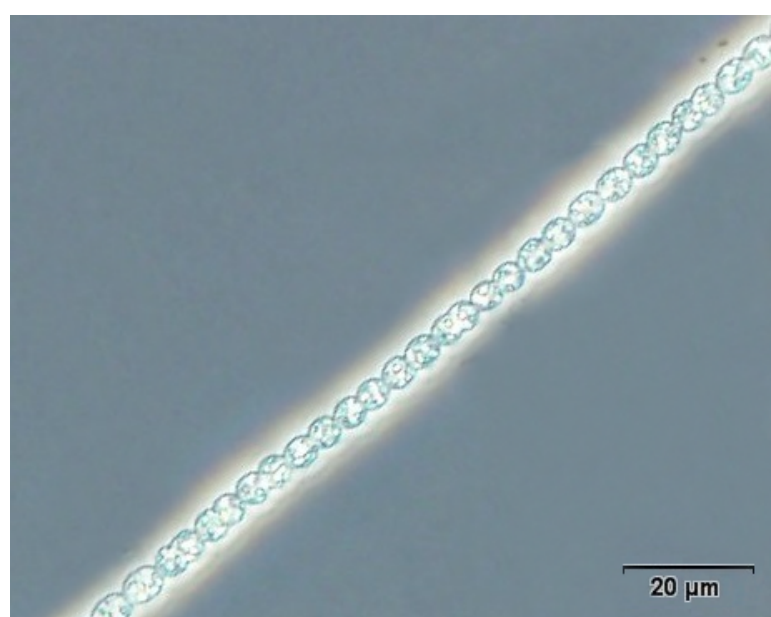

(b) A Anabaena circinalis Rabenhorst strain from (CSIRO ID: ACCR03) isolated from Murrumbidgee River, New South Wales, Australia.

Figure 1: Photographs of two strains of Anabaena circinalis Rabenhorst studied in this paper.

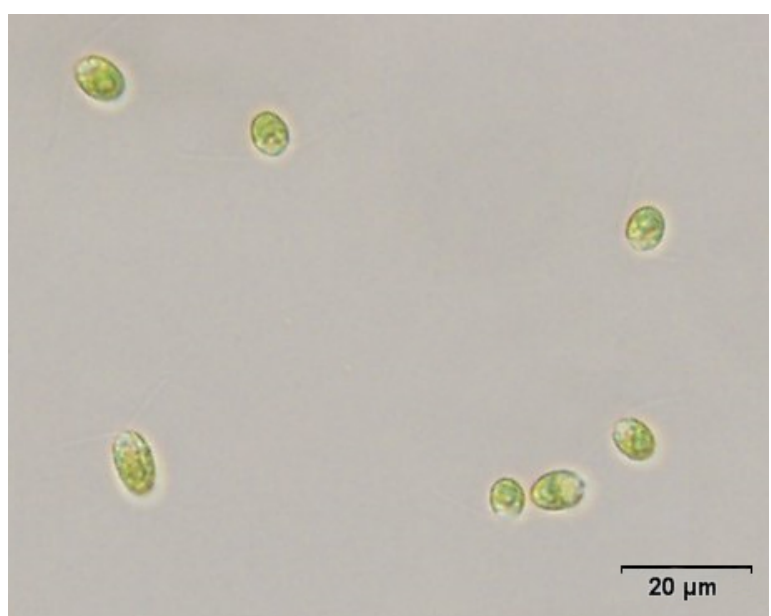

(a) A bright field image showing the details of the cells.

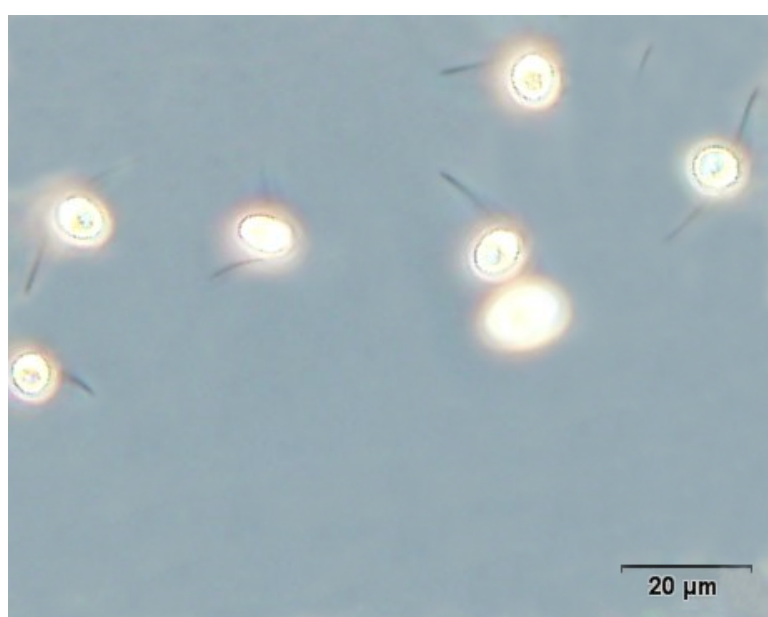

(b) A phase contrast image showing the flagella (the appendage used for swimming).

Figure 2: Photographs of the Dunaliella tertiolecta isolated from Port Phillip Bay, Victoria, Australia.

Freshwater species (A. circinalis) were grown in an MLA medium (provided by CSIRO), while the single marine species was grown in a modified "f-medium" ${ }^{1}$. Individual cultures were maintained in 
$25 \mathrm{~mL}$ Tissue Culture Flasks (TCFs) - canted neck, non-pyrogenic, sterile, polystyrene flasks (Corning Inc.). The cultures were grown at room temperature $\left(22^{\circ} \mathrm{C} \pm 2^{\circ} \mathrm{C}\right)$, under cool white fluorescent lights with a 13:11 light dark cycle and photon flux density of $\sim 40 \mu$ Einsteins.

Two strains of Anabaena circinalis Rabenhorst strains with CSIRO IDs of ACMR01 (figure 1a) and ACCR03 (figure 1b) were acquired from the CSIRO (Commonwealth Scientific and Industrial Research Organisation) in September 2014. The former was isolated from the Canning River, Perth, Western Australia, whereas the latter was isolated from the Murrumbidgee River, New South Wales, Australia. The Dunaliella tertiolecta (figure 2) was isolated from Port Phillip Bay, Victoria, Australia in December 2009 .

\section{Simulation of the control system to trap a target object}

The effect of the length, width and height of the constraints on the outlet channels on the ratio of the outlet flow rates and the position of the stagnation point can be studied using the analytical solution of the Navier-Stokes equations in rectangular channels. Here, we adopt the parametric study carried out by Tanyeri et al. ${ }^{2}$ to simulate the microfluidic trap and its control system. The simulation of the control system assists in predicting trajectory of a target object and ascertaining the possibility of the trapping at different conditions such as flow rate, initial position of the target and the trap time response.

Table 1 lists the dimensions and normalised parameters of the microfluidic device (fabricated in this work) used to simulate the control system.

Table 1: Specifications of the channel and its constraints and normalised parameters used in the simulation.

\begin{tabular}{ll||ll}
\hline $\begin{array}{l}\text { Channel and constraints } \\
\text { dimensions }\end{array}$ & $(\mu \mathrm{m})$ & Normalised dimensions & \\
\hline Channel width $(w)$ & 400 & & \\
Channel height $(h)$ & 40 & & \\
Outlet channel length $(L)$ & 7000 & & \\
Valve height $\left(h_{v}\right)$ & $($ variable) & $h_{v}^{*}=h_{v} / h$ & (variable) \\
Valve length $\left(l_{v}\right)$ & 400 & $l_{v}^{*}=l_{v} / L$ & 0.0571 \\
Fixed constriction width $\left(w_{c}\right)$ & 100 & $w_{c}^{*}=w_{c} / w$ & 0.25 \\
Fixed constriction length $\left(l_{c}\right)$ & 1800 & $l_{c}^{*}=l_{c} / L$ & 0.2571 \\
\hline
\end{tabular}

\subsection{Laminar flow in a rectangular microchannel}

The Navier-Stokes equations and boundary conditions for a rectangular channel with the height of $h$, width of $w$ and length of $L$ (with an aspect ratio of $\alpha_{r}=\frac{h}{w}$ and in our case $\alpha_{r}=0.1$ ), are given by ${ }^{3}$ :

$$
\begin{gathered}
\left(\frac{\partial^{2}}{\partial y^{2}}+\frac{\partial^{2}}{\partial z^{2}}\right) u=-\frac{\Delta P}{\nu L}, \\
\text { where }-\frac{w}{2} \leqslant y \leqslant \frac{w}{2} \text { and } 0 \leqslant z \leqslant h, \\
\text { Boundary Conditions: } u=0 \text { at }\left\{\begin{array}{l}
y= \pm \frac{w}{2} \\
z=0 \text { and } z=h
\end{array},\right.
\end{gathered}
$$

where the stream-wise velocity component $u(y, z)$ is a function of $y, z$ variables. Axes of the coordinate along the stream-wise (length), span-wise (width) and transverse (height) of the rectangular channel are represented by $x, y$ and $z$, respectively. The flow is assumed to be pressure-driven ( $\Delta P$ is the pressure difference over the distance L between the channel inlet and outlet), steady state and fully developed with no slip conditions on the walls. The analytical solution of equation 1 is provided as a Fourier series $\operatorname{expansion}^{3}$ :

$$
u(y, z)=\frac{4 h^{2} \Delta P}{\pi^{3} \nu L} \sum_{n, o d d}^{\infty} \frac{1}{n^{3}}\left[1-\frac{\cosh \left(n \pi \frac{y}{h}\right)}{\cosh \left(n \pi \frac{w}{2 h}\right)}\right] \sin \left(n \pi \frac{z}{h}\right)
$$


By integrating the velocity (equation 2) over the rectangular cross-section area, the volumetric flow rate can be expressed as ${ }^{3}$ :

$$
Q=2 \int_{0}^{w / 2} d y \int_{0}^{h} u(y, z) d z=\frac{h^{4} \Delta P}{12 \nu L \alpha}\left[1-\sum_{n, \text { odd }}^{\infty} \frac{192 \alpha}{(n \pi)^{5}} \tanh \left(\frac{n \pi}{2 \alpha}\right)\right] .
$$

\subsection{Flow resistance of the outlets of the cross-slot channel}

Based on the analogy with Ohm's law, the resistance of the laminar flow in a channel is defined $\operatorname{as}^{4}$ :

$$
R=\frac{\Delta P}{Q}
$$

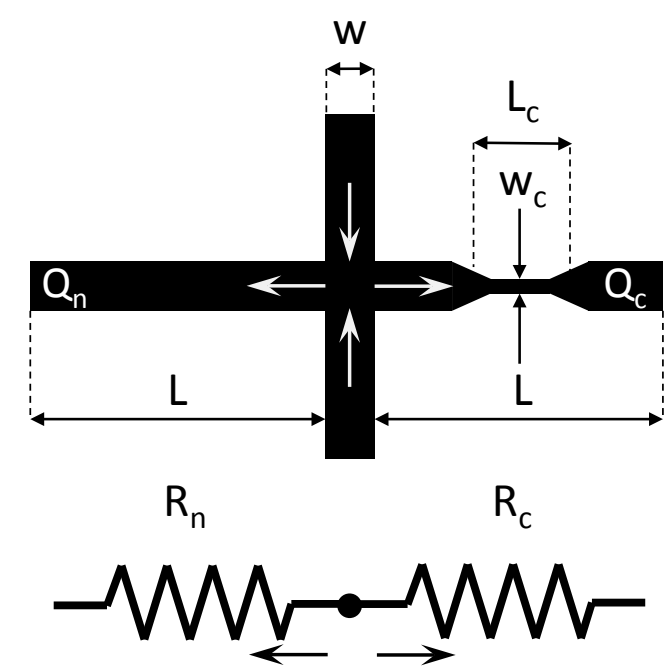

Figure 3: Schematic of the cross-slot junction with a fixed width constriction on the right-hand side outlet, and no constriction on left-hand side outlet. The width and the length of the constriction are shown by $w_{c}$ and $l_{c}$, respectively. The height of the constriction is the same as the channel height $(h)$. The outgoing flow rate from the constricted channel and its corresponding resistance are represented by $Q_{c}$ and $R_{c}$, respectively. Those of the channel with no constriction are shown by $Q_{n}$ and $R_{n}$, respectively.

Therefore, in the cross-junction shown in figure 3 , the ratio of the outlet flow rate with a fixed width constriction $\left(Q_{c}\right)$ to the total flow rate $\left(Q=Q_{n}+Q_{c}\right)$ can be stated as:

$$
\frac{Q_{c}}{Q}=\frac{\frac{\Delta P_{c}}{R_{c}}}{\frac{\Delta P_{c}}{R_{c}}+\frac{\Delta P_{n}}{R_{n}}}=\frac{\frac{1}{R_{c}}}{\frac{1}{R_{c}}+\frac{1}{R_{n}}}=\frac{R_{n}}{R_{n}+R_{c}}=\frac{1}{1+\frac{R_{c}}{R_{n}}},
$$

where $R_{n}$ and $R_{c}$ are the flow resistance of the channel with no constriction and with a fixed width constriction, respectively. Also it is assumed that the pressure drop in both outlets is the same $\left(\Delta P_{c}=\right.$ $\left.\Delta P_{n}\right)$. This assumption is correct if the length of the constriction is much smaller than the outlet length $\left(l_{c}<<L\right)$.

\subsection{The effect of the constriction width and length on the ratio of the flow rates through the outlet channels}

Using equations 3 and 4 , the resistance of the outlet channel with no constriction $\left(R_{n}\right.$ shown in the left outlet in figure 3) can be expressed as:

$$
R_{n}=\frac{12 \nu L \alpha}{h^{4}}\left[1-\sum_{n, o d d}^{\infty} \frac{192 \alpha}{(n \pi)^{5}} \tanh \left(\frac{n \pi}{2 \alpha}\right)\right]^{-1}
$$


If there is a constriction with the length and width of $l_{c}$ and $w_{c}$, respectively, and the same channel height $(h)$, the resistance of the whole constricted outlet channel (the right outlet with the length of $L$ in figure 3) using $\Delta P_{c}=\Delta P_{n}\left(1-l_{c}^{*}\right)+\Delta P_{c t r}$ is given by:

$$
R_{c}=R_{n}\left(1-l_{c}^{*}\right)+R_{c t r}
$$

where the resistance of the constriction part is represented by $R_{c t r}$ (the part of the right outlet, that has the length of $l_{c}$ ) and $l_{c}^{*}$ is the normalised constriction length (listed in table 1 ). Using equations 3 and $4, R_{c t r}$ can be stated as:

$$
R_{c t r}=\frac{12 \nu L \alpha}{h^{4}}\left(\frac{l_{c}^{*}}{w_{c}^{*}}\right)\left[1-\sum_{n, \text { odd }}^{\infty} \frac{192}{(n \pi)^{5}}\left(\frac{\alpha}{w_{c}^{*}}\right) \tanh \left(n \pi \frac{w_{c}^{*}}{2 \alpha}\right)\right]^{-1},
$$

where $w_{c}^{*}$ is the normalised constriction width (listed in table 1).

Using equations $5-8$, the variation of the relative flow rate in the constricted outlet channel versus variation of the length and width of the fixed constriction is shown in figure 4 . The marked point is the specification of the channel listed in table 1 .

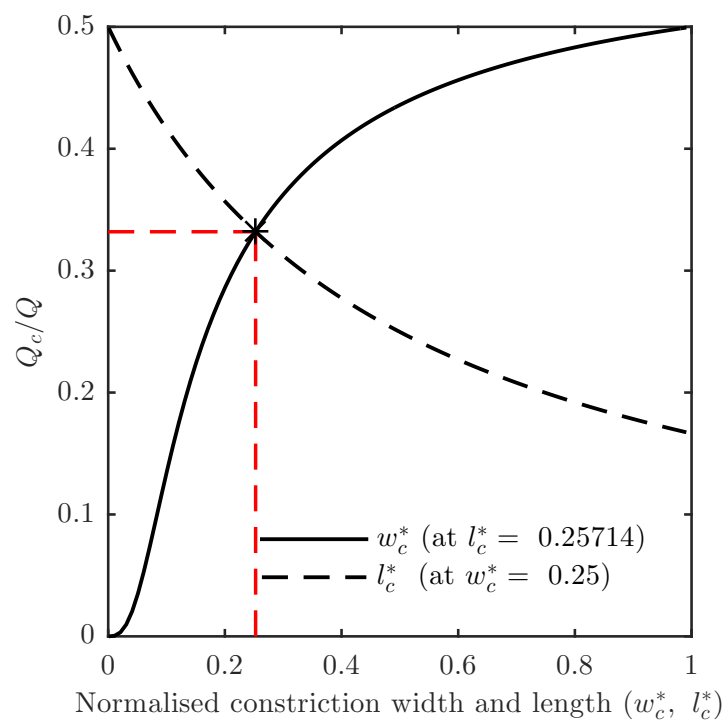

Figure 4: The effect of the normalised length $\left(l_{c}^{*}\right)$ and width $\left(w_{c}^{*}\right)$ of the fixed width constraint (in figure 3) on the relative flow rates.

\subsection{The effect of the constriction height (membrane valve) on the ratio of the flow rates through the outlet channels}

The same analogy for calculating the resistance of the outlet with the fixed width constriction is applied to compute the resistance of the outlet with variable height constriction $\left(R_{v}\right)$ that is shown in figure 5 . Therefore, $R_{v}$ is given by:

$$
R_{v}=R_{n}\left(1-l_{v}^{*}\right)+R_{v a l},
$$

where $R_{v a l}$ is the resistance of the constrictor section with the length of $l_{v}$ and height of $h_{v}$ (the red feature in figure 5 ). Using equations 3 and $4, R_{v a l}$ is given by:

$$
R_{v a l}=\frac{12 \nu L \alpha}{h^{4}}\left(\frac{l_{v}^{*}}{h_{v}^{* 3}}\right)\left[1-\sum_{n, o d d}^{\infty} \frac{192 \alpha h_{v}^{*}}{(n \pi)^{5}} \tanh \left(\frac{n \pi}{2 \alpha h_{v}^{*}}\right)\right]^{-1}
$$

where $h_{v}^{*}, l_{v}^{*}$ are normalised constriction height and length (listed in table 1), respectively.

Therefore, in the cross-junction shown in figure 5, (using a similar procedure used to derive equation $5)$ the ratio of the outlet flow rate with a variable constriction $\left(Q_{v}\right)$ to the total flow rate $\left(Q=Q_{v}+Q_{c}\right)$ can be stated as: 

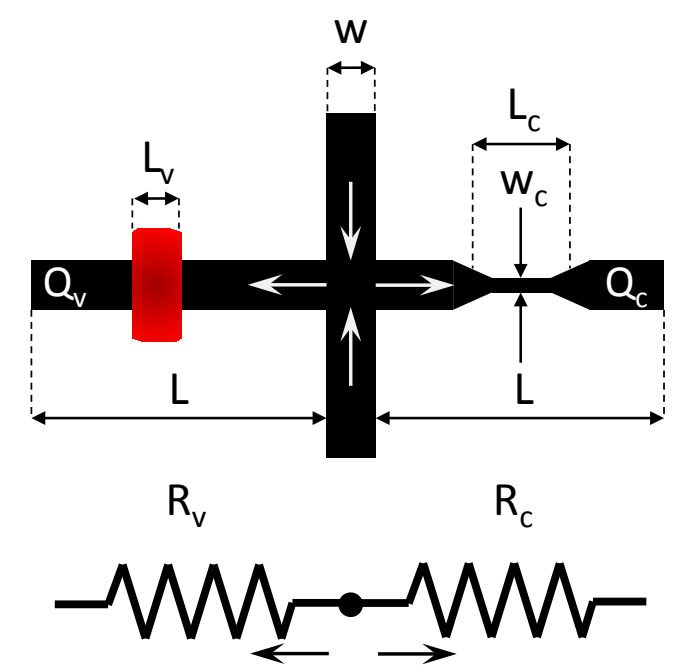

Figure 5: Schematic of the cross-slot junction with a fixed width constriction on the right-hand side outlet and height constriction on the left-hand side outlet. The height constriction is shown by a red channel that represents the existence of the membrane valve, capable of adjusting the channel height. The width and the length of the width constrictions are shown by $w_{c}$ and $l_{c}$, respectively. The outgoing flow rate from the width constricted channel and its corresponding resistance are represented by $Q_{c}$ and $R_{c}$, respectively. The length of the height constriction on the left-hand side outlet is shown by $l_{v}$ that is equal to the width of the membrane valve. The outgoing flow rate from the height constricted channel and its corresponding resistance are represented by $Q_{v}$ and $R_{v}$, respectively.

$$
\frac{Q_{v}}{Q}=\frac{1}{1+\frac{R_{v}}{R_{c}}} .
$$

Using equations 7-11 the effect of the variable height constriction on the relative flow rate with and without the fixed width constriction (on the other outlet) is shown in figure 6. Comparing these two curves, it is confirmed that the existence of both fixed and variable constraints on both outlets resulted in obtaining a wider range of flow rate variation (compared to the case that there is only variable height constraint). Achieving a wider range of flow rate variation enables repositioning of the stagnation point in the wider range. In other words, the fixed width constraint works as an offset for the ratio of the flow rate and the position of stagnation point in the cross-junction. Therefore, an initial pressure in the control channel is required to deflect the membrane and equalise the outlet flow rates to place the stagnation point at the centre. Therefore, the possibility of trapping objects in a microfluidic trap with two constraints (fixed and variable) is higher than that with only one variable constraint.

In reality the membrane is only able to partially block the channel to a certain height. The grey region in figure 6 shows the region that cannot be reached in experiments.

\subsection{The effect of the relative flow rate on the position of the stagnation point}

To find the relation between the outlet flow rates and the position of the stagnation point, an arbitrary position of the stagnation point $\left(Y_{o}\right)$, shown in figure $7 \mathrm{a}$, is considered. Then, by integrating the velocity field corresponding to each outlet, the flow rate of each outlet is yielded as the function of the stagnation point:

$$
Q_{v}=\int_{-w / 2}^{Y_{o}} d y \int_{0}^{h} u(y, z) d z, \quad Q_{c}=\int_{Y_{o}}^{w / 2} d y \int_{0}^{h} u(y, z) d z
$$

Therefore, the ratio of the outlet flow rates as a function of the stagnation point position is obtained by $^{5}$ : 


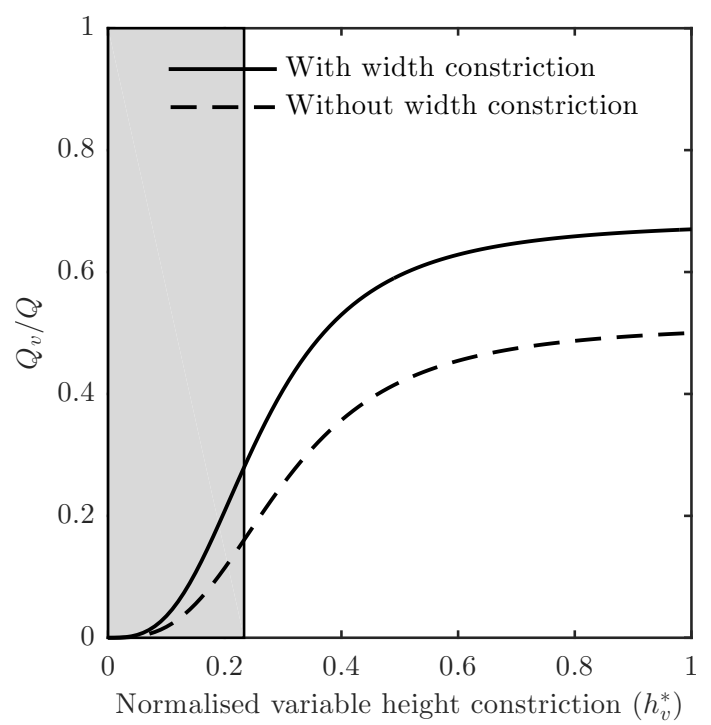

Figure 6: The effect of the normalised height $\left(h_{v}^{*}\right)($ the right outlet in figure 5$)$ on the relative flow rate. The solid line shows the relative flow rate variations when there is a fixed constraint on the other outlet (the outlet in figure 5). The dashed line shows the variation with the absence of the fixed constriction on the other outlet. In accordance with Tanyeri et al. ${ }^{2}$, the grey region is not achievable as the membrane valve cannot block the cross-section more than a certain amount.

$$
\frac{Q_{v}}{Q}\left(Y_{o}^{*}\right)=\frac{Y_{o}^{*}+\frac{1}{2}\left\{1-\sum_{n, \text { odd }}^{\infty} \frac{192 \alpha}{(n \pi)^{5}}\left[\frac{\sinh \left(n \pi \frac{Y_{o}^{*}}{\alpha}\right)}{\cosh \left(\frac{n \pi}{2 \alpha}\right)}+\tanh \left(\frac{n \pi}{2 \alpha}\right)\right]\right\}}{1-\sum_{n, \text { odd }}^{\infty} \frac{192 \alpha}{(n \pi)^{5}} \tanh \left(\frac{n \pi}{2 \alpha}\right)} .
$$

where $Y_{o}^{*}=Y_{o} / w$ is the normalised stagnation point position along $y$-direction (extensional axis). Figure $7 \mathrm{~b}$ shows the linear behaviour of the stagnation point position variations at different relative flow rates. As previously mentioned, in experiments the membrane is only able to partially block the channel to a certain height. The experimentally obtainable variation of the normalised channel height $\left(h_{v}^{*}\right)$ is replotted in figure $7 \mathrm{~b}$ to show the region where the stagnation point can be displaced (the region between the two red dashed lines). Computing $h_{v}^{*}$ as a function of $Y_{o}^{*}$ (the dashed blue line) was carried out by obtaining the $Q_{v} / Q$ as a function of $h_{v}^{*}$ from figure 6 and substituting them in equation 13 .

\subsection{Estimation of the required pressure to reposition the stagnation point}

To estimate the required gas pressure in the control channel to reposition the stagnation point, the relation between the pressure and the height of the variable constraint is required. In principle, it is possible to analyticity calculate the deformation of the membrane using the governing equations of the deflection of the uniformly loaded flat plate. However, it is challenging to measure the stiffness of the PDMS experimentally in order to compute the deflection. In this simulation, we adopted the valve response data (shown in figure 8a) by Tanyeri et al. ${ }^{5}$, i.e. the pressure in the control channel at different percentages of the valve opening $\left(h_{v}^{*}\right)$. However, in the experiments conducted in this paper, the final calibration curve was obtained experimentally. For experimentally determining the final calibration curve, it is not necessary to have valve response data (figure 8a), as it is possible to directly obtain the stagnation point positions at different pressures in the membrane valve. Therefore, valve response data by Tanyeri et al. ${ }^{5}$ is only used for the simulation.

For the membrane thickness of $66 \mu \mathrm{m}$ and the ratio of the PDMS base to cross-linker of 1:15 the data is shown in figure $8 \mathrm{a}^{5}$. A linear response can be seen where the valve opening is more than $30 \%$.

Eventually, using the data provided in figure $7\left(h_{v}^{*}\right.$ as a function of $\left.Y_{o}^{*}\right)$ and figure $8 \mathrm{a}(P$ as a function of $h_{v}^{*}$ ), the variations of the stagnation point position at different control channel pressures $\left(Y_{o}^{*}\right.$ as a function of $P$ ) were obtained and are shown in figure $8 \mathrm{~b}$. This graph is used as the calibration curve of the microfluidic trap. 


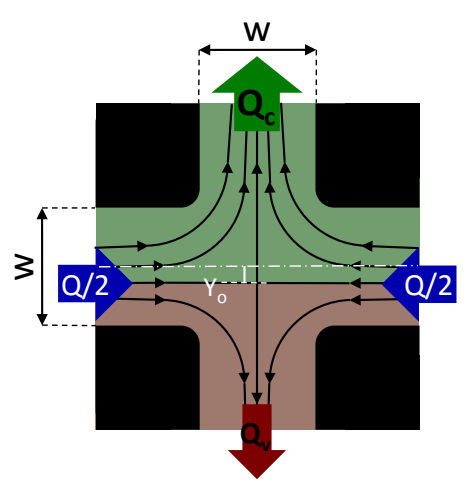

(a) An arbitrary stagnation point position in the cross-junction, where the inlet flow $(Q / 2)$ bifurcates into the two outlets, $Q_{c}$ and $Q_{v}$. The distance between the centre line of the channel and the stagnation point along the $y$-direction is $Y_{o}$

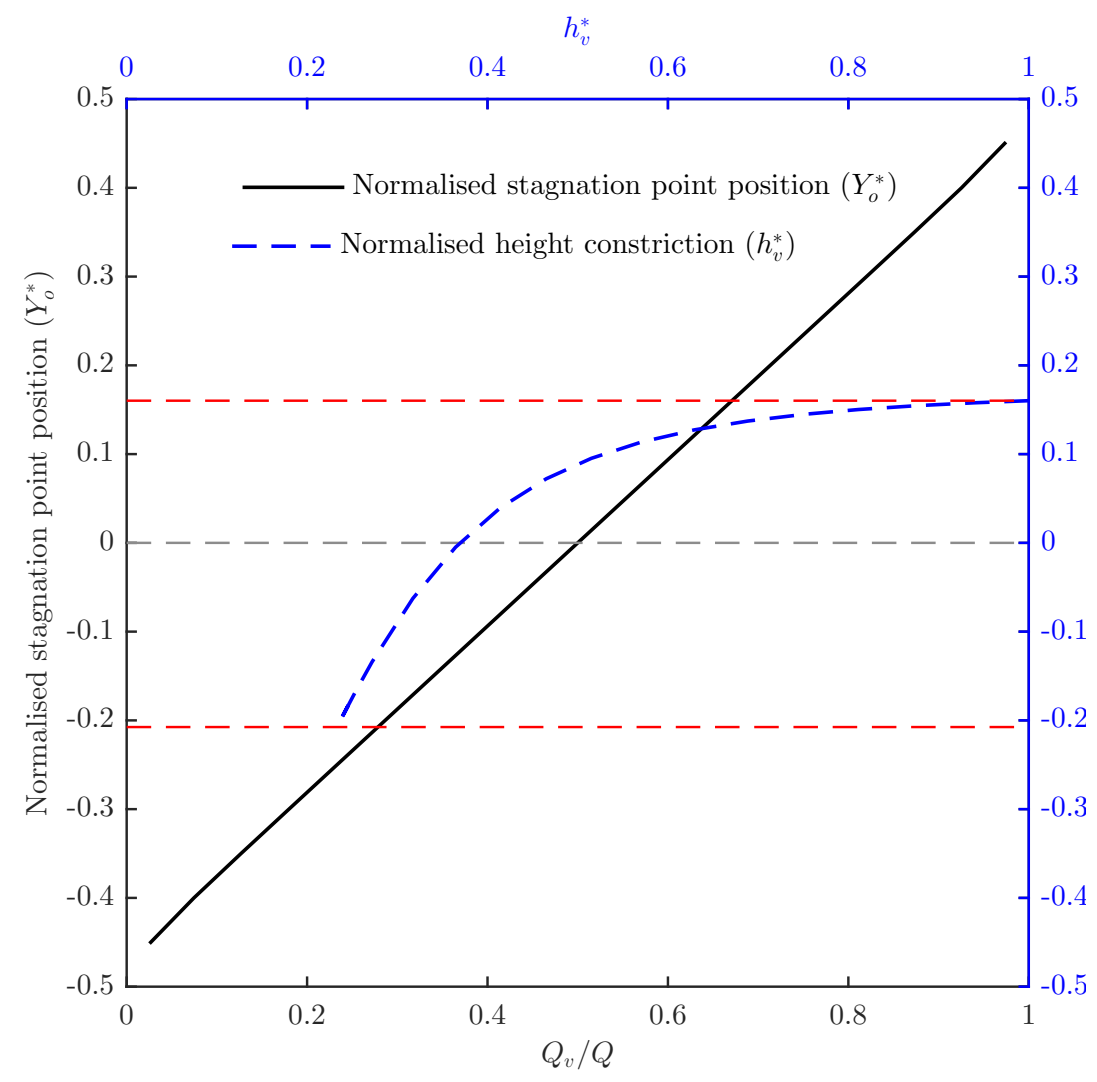

(b) The black line (with the corresponding black left and bottom axes) shows the variation of the stagnation point at various relative flow rates. The dashed blue line (with the corresponding blue right and top axes) indicates the region of height variation that is experimentally achievable (previously shown in the white region in figure 6). Computing $h_{v}^{*}$ as a function of $Y_{o}^{*}$ (the dashed blue line) was carried out by obtaining the $Q_{v} / Q$ from figure 6 and substituting them in equation 13 , thereby indicating the region (between the two red lines) where the stagnation point can be experimentally displaced.

Figure 7: The effect of the relative flow rate $\left(Q_{v} / Q\right)$ and the normalised height of the variable constriction $\left(h_{v}^{*}\right)$ on the normalised stagnation point position $\left(Y_{o}^{*}\right)$.

\subsection{Prediction of the trajectory of a target object in the microfluidic trap}

Using the estimated calibration curve (figure $8 \mathrm{~b}$ ) the trajectory of the target that enters the cross-junction from an arbitrary location can be predicted. Based on the centroid position of the target and using the equation

$$
P_{v a l}=P_{t c}+K_{P} K_{C} e_{t t}
$$

the gas pressure in the control layer is updated, thereby displacing the stagnation point to a new location.

As mentioned in the manuscript, for using equation 14 , the conversion factor $\left(K_{C}\right)$ is required. This factor $\left(K_{C}\right)$ is the slope of the fitted linear line in figure $8 \mathrm{~b}$. The equation of the fitted line $\left(Y_{o}^{*}=-0.0459 P_{v a l}+0.48238\right)$ is used to determine the position of the stagnation point. In other words, the stagnation point position is determined using (a linear interpolation of) the calibration curve.

In this simulation, the stagnation point is repositioned at $f_{\text {sys }}=30 \mathrm{~Hz}$, which is the frequency of the control system. In an experiment, control system delay time $\Delta t_{\text {delay }}=f_{\text {sys }}^{-1}$ is the period of time during which inline image processing is performed to detect the target plus the period of time the pressure valve operates to regulate the pressure. The lower the delay time, the greater the possibility of trapping objects at higher strain rates.

Figure 9 shows the trajectory of the targets in the cross-region during which the trapping was simulated. Normalised $x$ and $y$ axes are shown as $x^{*}=x / w, y^{*}=y / w$ in this figure. As can be seen, when the strain rate or $y_{t 0}$ (the initial distance along the extensional axis between the target and the centre line of the channel) increase, the trap is not able to confine the target, e.g. $\left(\gamma=20 \mathrm{~s}^{-1}, y_{t 0}=-10 \mu \mathrm{m}\right)$ 


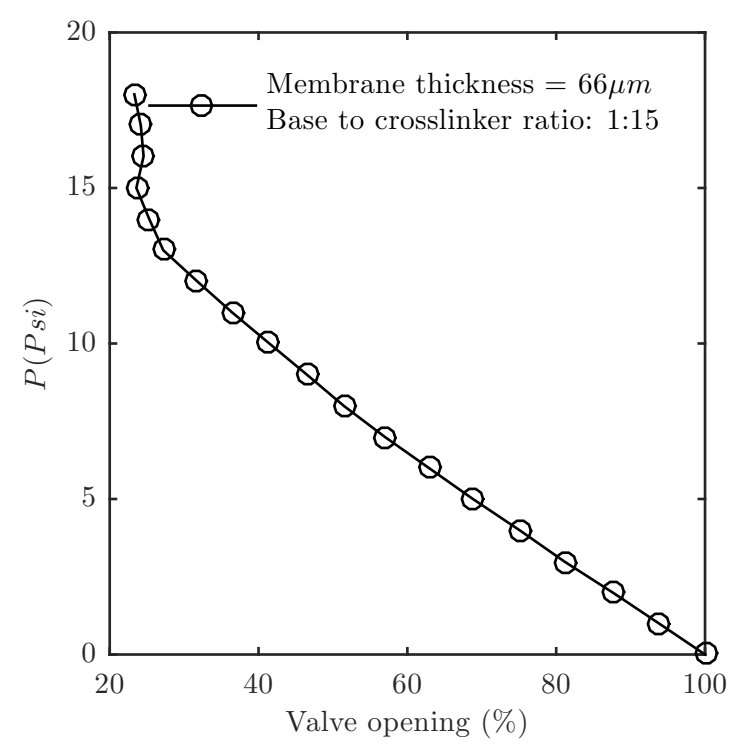

(a) The percentage of the valve opening at different gas pressures in the control layer for a PDMS membrane with a thickness of $66 \mu \mathrm{m}$ and a ratio of the base to cross-linker of $1: 15^{5}$.

Figure 8: Valve response and the variation of the stagnation point position at the different pressures on the membrane valve.

and $\left(\gamma=15 s^{-1}, y_{t 0}=25 \mu \mathrm{m}\right)$. However, if in these two cases either the strain rate or the $y_{t 0}$ decrease, it is possible to trap the targets i.e. $\left(\gamma=15 \mathrm{~s}^{-1}, y_{t 0}=10 \mu \mathrm{m}\right)$ and $\left(\gamma=2.5 \mathrm{~s}^{-1}, y_{t 0}=25 \mu \mathrm{m}\right)$. In these two cases, although the targets experienced some fluctuations (shown in figure $9 \mathrm{~b}$ ), both cases confirm the convergence of the target at the trap centre.

In figure 10, the required pressure and pressure differences in the control channel are shown. The pressure changes for these targets in figure 9 were between about 4 to 13 psi and the maximum pressure difference required to trap objects was about 3 psi. This information assists in selecting the electronic pressure valve for the experiments.

Considering the data in this simulation, it can be confirmed that trapping objects entering at $(-20<$ $\left.y_{t 0}<20\right)$ and the strain rate below $10 \mathrm{~s}^{-1}$ is possible. Although we intend to expose high strain rates to the microorganisms, it is not initially necessary to deliver the fluid at a high flow/strain rate. In experiments, the pressure regulator responds faster for smaller pressure changes, and this is typically more relevant after the initial confinement of a target (shown in figure 10). Therefore, it is possible to confine a target at a low flow rate and then gradually increase the flow rate/strain rate. In this case, smaller pressure changes are required to bring the target back to the trap centre when the target is displaced.

\section{Analytical solution of the flow over an ellipsoidal object im- mersed in a creeping straining flow}

Here, we adopt the analytical solution of the flow over an ellipsoidal object immersed in a creeping flow, wherein each component of the velocity field is a linear function of the Cartesian coordinates, $x, y$ and $z$, presented by Jeffery ${ }^{6}$. We simplify the solution to the pure straining flow around an ellipsoid.

\subsection{Confocal ellipsoidal coordinate}

The confocal ellipsoidal coordinate is represented by ${ }^{7}$ :

$$
\frac{x^{2}}{a^{2}+\lambda}+\frac{y^{2}}{b^{2}+\lambda}+\frac{z^{2}}{c^{2}+\lambda}=1
$$




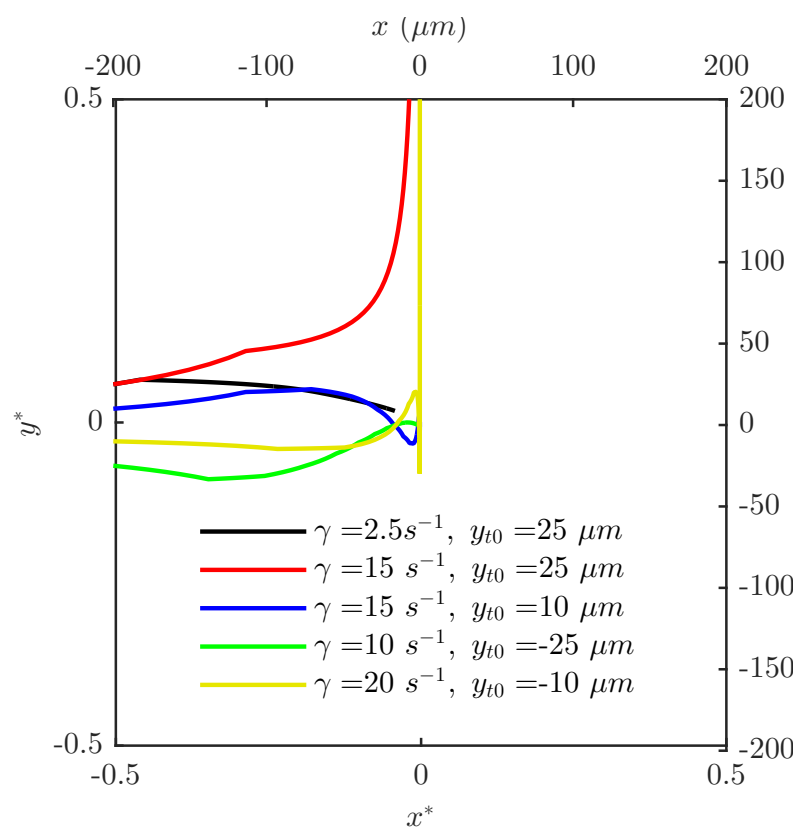

(a) Trajectory of the target objects entered the cross-region at different initial locations and the strain rates of the flow.

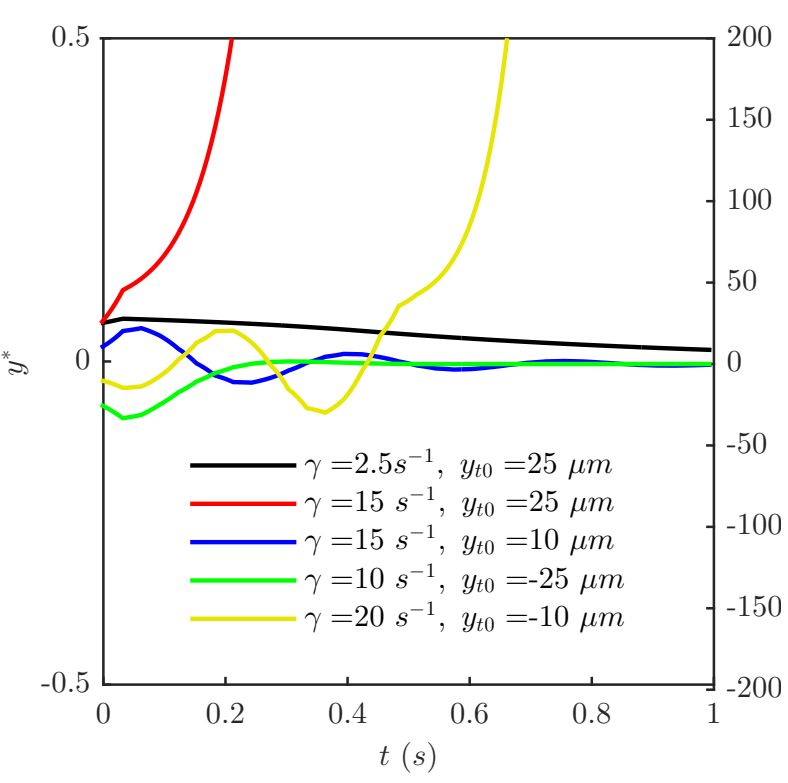

(b) Variations of the locations of the target objects along the extensional axis entered the cross-region at different initial locations and the strain rates of the flow.

Figure 9: Predicted trajectory of five targets that entered the cross-region at different locations and strain rates during trapping.

where $a, b$ and $c$ are the length of the semi-principle axes of the ellipsoid along the $x, y$ and $z$ of the Cartesian coordinate, respectively. Also $\lambda$ is a constant that produces the surface of confocal ellipsoids in the ellipsoidal coordinate. The variables $\Delta$ and $\Upsilon$ used in the confocal ellipsoidal coordinate are defined as:

$$
\begin{aligned}
& \Delta=\sqrt{\left(a^{2}+\lambda\right)+\left(b^{2}+\lambda\right)+\left(c^{2}+\lambda\right)}, \\
& \Upsilon=\left(\frac{x^{2}}{a^{2}+\lambda}+\frac{y^{2}}{b^{2}+\lambda}+\frac{z^{2}}{c^{2}+\lambda}\right)^{-\frac{1}{2}} .
\end{aligned}
$$

The auxiliary variables in confocal ellipsoidal coordinates that are defined through the Jacobi elliptic integral transforms, are given by:

$$
\begin{aligned}
& \alpha=\int_{\lambda}^{\infty} \frac{d \lambda}{\left(a^{2}+\lambda\right) \Delta}, \alpha^{\prime}=\int_{\lambda}^{\infty} \frac{d \lambda}{\left(b^{2}+\lambda\right)\left(c^{2}+\lambda\right) \Delta}, \alpha^{\prime \prime}=\int_{\lambda}^{\infty} \frac{\lambda d \lambda}{\left(b^{2}+\lambda\right)\left(c^{2}+\lambda\right) \Delta}, \\
& \beta=\int_{\lambda}^{\infty} \frac{d \lambda}{\left(b^{2}+\lambda\right) \Delta}, \beta^{\prime}=\int_{\lambda}^{\infty} \frac{d \lambda}{\left(a^{2}+\lambda\right)\left(c^{2}+\lambda\right) \Delta}, \beta^{\prime \prime}=\int_{\lambda}^{\infty} \frac{\lambda d \lambda}{\left(a^{2}+\lambda\right)\left(c^{2}+\lambda\right) \Delta}, \\
& \Gamma=\int_{\lambda}^{\infty} \frac{d \lambda}{\left(c^{2}+\lambda\right) \Delta}, \Gamma^{\prime}=\int_{\lambda}^{\infty} \frac{d \lambda}{\left(a^{2}+\lambda\right)\left(b^{2}+\lambda\right) \Delta}, \Gamma^{\prime \prime}=\int_{\lambda}^{\infty} \frac{\lambda d \lambda}{\left(a^{2}+\lambda\right)\left(b^{2}+\lambda\right) \Delta} .
\end{aligned}
$$

\subsection{Velocity field around an ellipsoid immersed in the pure straining creeping flow}

Jeffery ${ }^{6}$ solved the Stokes equations of the creeping flow around the ellipsoidal particle, where each undisturbed velocity component away from the vicinity of the particle is linearly a function of $x, y$ and $z$ 


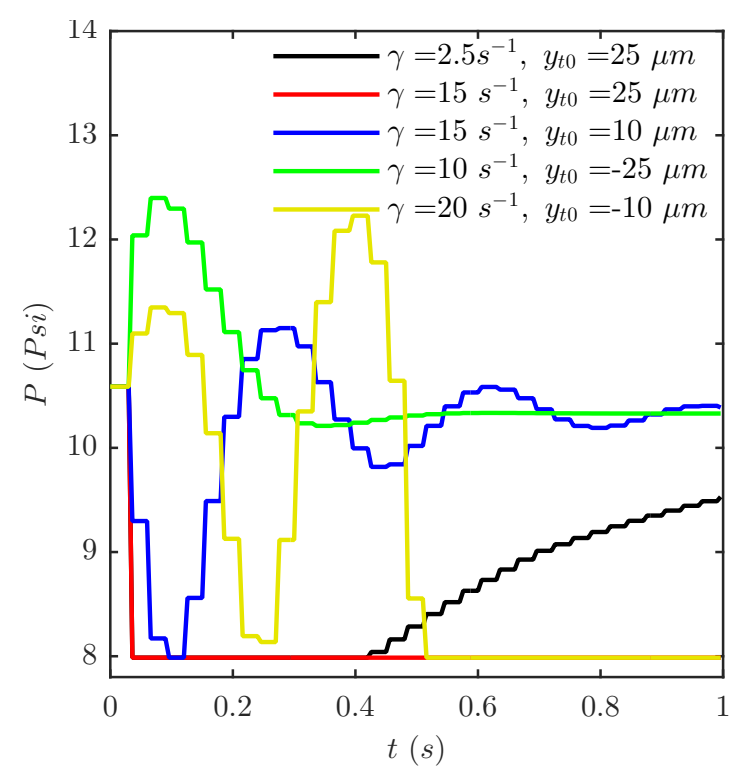

(a) The pressure in the control channel to trap the targets in figure 9.

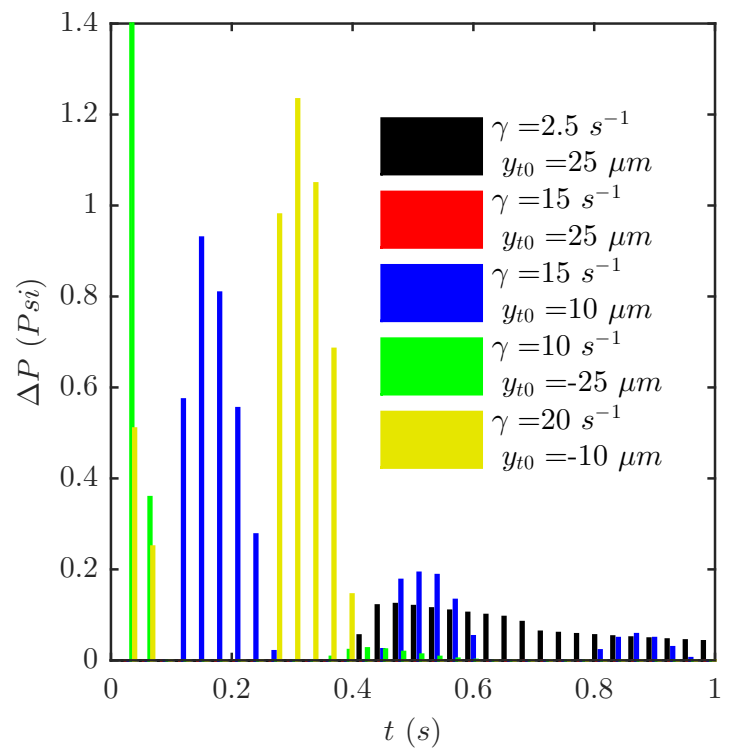

(b) The pressure difference in the control channel to trap the targets in figure 9 .

Figure 10: Required pressures and pressure differences for trapping the five targets in figure 9.

$$
\begin{aligned}
& u_{0}=a_{0} x+h_{0} y+g_{0} z+\eta_{0} z-\zeta_{0} y, \\
& v_{0}=h_{0} x+b_{0} y+f_{0} z+\zeta_{0} x-\xi_{0} z, \\
& w_{0}=g_{0} x+f_{0} y+c_{0} z+\xi_{0} y-\eta_{0} x,
\end{aligned}
$$

where all the coefficients of $x, y$ and $z$ are constants and the components of flow distortion and rotation. In the case of pure straining flow these equations are simplified to:

$$
\begin{aligned}
& u_{0}=a_{0} x, \\
& v_{0}=b_{0} y,
\end{aligned}
$$

where $a_{0}=\gamma$ and $b_{0}=-\gamma$, and $\gamma$ represents the strain rate of the flow.

By simplifying Jeffrey's solution according to equation 19, the velocity components $u, v$ and $w$ (as functions of $x, y, z, \lambda$ and the auxiliary variables in equations 16 and 17) around an ellipsoid (with the semi-principle axes of $a, b$ and $c$ ) in the straining flow are given by:

$$
\begin{gathered}
u=\left[\gamma+\Gamma^{\prime} W-\beta^{\prime} V-2(\alpha+\beta+\Gamma) A\right] x \\
-\frac{2 \Upsilon^{2}}{\left(a^{2}+\lambda\right) \Delta}\left[\frac{W-2\left(a^{2}+\lambda\right) A+2\left(b^{2}+\lambda\right) B}{\left(b^{2}+\lambda\right)^{2}} x y^{2}\right. \\
\left.-\frac{V-2\left(c^{2}+\lambda\right) C+2\left(a^{2}+\lambda\right) A}{\left(c^{2}+\lambda\right)^{2}} x z^{2}\right] \\
v=\left[-\gamma+\alpha^{\prime} U-\Gamma^{\prime} W-2(\alpha+\beta+\Gamma) B\right] y \\
-\frac{2 \Upsilon^{2}}{\left(b^{2}+\lambda\right) \Delta}\left[\frac{U-2\left(b^{2}+\lambda\right) B+2\left(c^{2}+\lambda\right) C}{\left(c^{2}+\lambda\right)^{2}} y z^{2}\right. \\
\left.-\frac{W-2\left(a^{2}+\lambda\right) A+2\left(b^{2}+\lambda\right) B}{\left(a^{2}+\lambda\right)^{2}} y x^{2}\right]
\end{gathered}
$$


and

$$
\begin{gathered}
w=\left[\beta^{\prime} V-\alpha^{\prime} U-2(\alpha+\beta+\Gamma) C\right] z \\
-\frac{2 \Upsilon^{2}}{\left(c^{2}+\lambda\right) \Delta}\left[\frac{V-2\left(c^{2}+\lambda\right) C+2\left(a^{2}+\lambda\right) A}{\left(a^{2}+\lambda\right)^{2}} z x^{2}\right. \\
\left.-\frac{U-2\left(b^{2}+\lambda\right) B+2\left(c^{2}+\lambda\right) C}{\left(b^{2}+\lambda\right)^{2}} z y^{2}\right],
\end{gathered}
$$

where $A, B, C, U, V$ and $W$ are given by:

$$
\begin{aligned}
A=\frac{1}{6}\left(\frac{2 \alpha_{0}^{\prime \prime} \gamma+\beta_{0}^{\prime \prime} \gamma}{\beta_{0}^{\prime \prime} \Gamma_{0}^{\prime \prime}+\Gamma_{0}^{\prime \prime} \alpha_{0}^{\prime \prime}+\alpha_{0}^{\prime \prime} \beta_{0}^{\prime \prime}}\right), & U=2 b^{2} B-2 c^{2} C, \\
B=\frac{1}{6}\left(\frac{-2 \beta_{0}^{\prime \prime} \gamma-\alpha_{0}^{\prime \prime} \gamma}{\beta_{0}^{\prime \prime} \Gamma_{0}^{\prime \prime}+\Gamma_{0}^{\prime \prime} \alpha_{0}^{\prime \prime}+\alpha_{0}^{\prime \prime} \beta_{0}^{\prime \prime}}\right), & V=2 c^{2} C-2 a^{2} A, \\
C=\frac{1}{6}\left(\frac{-\alpha_{0}^{\prime \prime} \gamma+\beta_{0}^{\prime \prime} \gamma}{\beta_{0}^{\prime \prime} \Gamma_{0}^{\prime \prime}+\Gamma_{0}^{\prime \prime} \alpha_{0}^{\prime \prime}+\alpha_{0}^{\prime \prime} \beta_{0}^{\prime \prime}}\right), & W=2 a^{2} A-2 b^{2} B .
\end{aligned}
$$

To obtain a velocity data-point located at $x, y$ and $z$, firstly $\lambda$ is obtained from equation 15 , which is a third-order equation. By substituting $\lambda$ into equation 16, $\Delta$ and $\Upsilon$ are determined. Then, all the nine integrals stated in equation 17 are solved numerically to compute the other ellipsoidal coordinate axillary variables. The parameters given in equation 23 are obtained using the axillary variables. Finally, by substituting the axillary variables (equations 16 and 17) and other parameters (equation 23) into equations 20,21 and 22 , the velocity components are determined.

\section{References}

[1] R. R. Guillard and J. H. Ryther, Can. J. Microbiol., 1962, 8, 229-239.

[2] M. Tanyeri, M. Ranka, N. Sittipolkul and C. M. Schroeder, Lab Chip, 2011, 11, 1786-1794.

[3] H. Bruus, Theoretical microfluidics, New York: Oxford University Press, 2008.

[4] A. Akers, M. Gassman and R. Smith, Hydraulic power system analysis, CRC press, 2006.

[5] M. Tanyeri, E. M. Johnson-Chavarria and C. M. Schroeder, Appl. Phys. Lett., 2010, 96, 224101.

[6] G. B. Jeffery, 1922, 102, 161-179.

[7] H. Feshbach and P. M. Morse, Methods of theoretical physics, McGraw-Hill Interamericana, 1953. 


\section{University Library}

\section{- M M N E R VA A gateway to Melbourne's research publications}

Minerva Access is the Institutional Repository of The University of Melbourne

Author/s:

Akbaridoust, F;Philip, J;Hill, DRA;Marusic, I

Title:

Simultaneous micro-PIV measurements and real-time control trapping in a cross-slot channel

Date:

2018-12-01

Citation:

Akbaridoust, F., Philip, J., Hill, D. R. A. \& Marusic, I. (2018). Simultaneous micro-PIV measurements and real-time control trapping in a cross-slot channel. Experiments in Fluids, 59 (12), https://doi.org/10.1007/s00348-018-2637-6.

Persistent Link:

http://hdl.handle.net/11343/282708 